\title{
Ruxolitinib combined with vorinostat suppresses tumor growth and alters metabolic phenotype in hematological diseases
}

\author{
Monica Civallero ${ }^{1}$, Maria Cosenza ${ }^{1}$, Samantha Pozzi ${ }^{1}$ and Stefano Sacchi ${ }^{1}$ \\ ${ }^{1}$ Department of Diagnostic, Clinical, and Public Health Medicine, University of Modena and Reggio Emilia, Modena, Italy \\ Correspondence to: Monica Civallero, email: mcivallero@unimo.it \\ Keywords: ruxolitinib, vorinostat, apoptosis, metabolism, cancer therapy
}

Received: July 28, $2017 \quad$ Accepted: September 24, $2017 \quad$ Published: October 23, 2017

Copyright: Civallero et al. This is an open-access article distributed under the terms of the Creative Commons Attribution License 3.0 (CC BY 3.0), which permits unrestricted use, distribution, and reproduction in any medium, provided the original author and source are credited.

\section{ABSTRACT}

JAK-2 dysregulation plays an important role as an oncogenic driver, and is thus a promising therapeutic target in hematological malignancies. Ruxolitinib is a pyrrolo[2.3-d]pyrimidine derivative with inhibitory activity against JAK1 and JAK2, moderate activity against TYK2, and minor activity against JAK3. Vorinostat is an HDAC inhibitor that reduces JAK-2 expression, thus affecting JAK-2 mRNA expression and increasing JAK-2 proteasomal deterioration. Here we hypothesized that the combination of ruxolitinib and vorinostat could have synergistic effects against hematological disease. We tested combinations of low doses of ruxolitinib and vorinostat in 12 cell lines, and observed highly synergistic cytotoxic action in six cell lines, which was maintained for up to $120 \mathrm{~h}$ in the presence of stromal cells. The sensitivity of the six cell lines may be explained by the broad effects of the drug combination, which can affect various targets. Treatment with the combination of ruxolitinib and vorinostat appeared to induce a possible reversal of the Warburg effect, with associated ROS production, apoptotic events, and growth inhibition. Decreased glucose metabolism may have markedly sensitized the six more susceptible cell lines to combined treatment. Therapeutic inhibition of the JAK/STAT pathway seems to offer substantial anti-tumor benefit, and combined therapy with ruxolitinib and vorinostat may represent a promising novel therapeutic modality for hematological neoplasms.

\section{INTRODUCTION}

During hematopoietic ontogenesis, cytokines play key roles by initiating the intracellular signals that govern cell fate decisions, such as proliferation and differentiation. Most cytokine receptors lose intrinsic kinase activity and, therefore, often employ Janus kinase (JAK) as a signaling intermediate to facilitate downstream signaling. Receptorrelated non-receptor tyrosine kinases, such as JAK1, JAK2, JAK3, and TYK2, are triggered after cytotoxic receptor activation [1]. JAK activation determines the phosphorylation of STAT transcription factors, including STAT1, STAT2, STAT3, STAT4, STAT5A, STAT5B, and STAT6. Following activation, STAT complexes translocate to the nucleus, bind DNA, and begin transcription $[2,3]$. Dysregulation of JAK/STAT pathways can cause hematological illnesses and immunodeficiency disorders, and is implicated in the pathogenesis of some solid tumors [4]. Hematologic malignancies exhibit abnormal activation of JAK2 signaling [5]. The JAK2V617F mutation has been identified in different patients with neoplasms, supporting the development of JAK inhibitors to specifically target JAK signaling [6].

Ruxolitinib (INCB018424) is an orally administered, biologically available pyrrolo[2.3-d]pyrimidine derivative that shows inhibitory activity against JAK1 and JAK2, moderate activity against TYK2, and minor activity against JAK3 [7, 8]. Clinical trial results indicate a mechanism of action in which anti-JAK-STAT action mediates down-regulation of inflammatory cytokine activity $[9,10]$. Ruxolitinib is approved by the US Food and Drug Administration (FDA) for use in myelofibrosis and polycythemia Vera, and is indicated for treatment of various solid tumors (breast, pancreatic, colorectal, head 
and neck, and prostate) and hematologic illnesses (CLL, ALL, AML, CML, and NSCLC) [11-14]. However, ruxolitinib does not appreciably reduce allele burden, and does not produce histologic or molecular cytogenetic remission in hematologic malignancies [15]. Furthermore, ruxolitinib resistance develops following chronic drug exposure [16], highlighting a clear need for combined therapies.

Histone deacetylase (HDAC) inhibitors are a promising group of therapeutic agents for various malignancies [17, 18]. Histone modifications may alter the expression and regulation of suppressor of cytokine signaling proteins (SOCS), a family of genes involved in JAK2-STAT3 signaling pathway inhibition [19, 20]. In October 2006, the FDA approved the HDAC inhibitor vorinostat for the treatment of advanced cutaneous T-cell lymphoma [21-23]. Most cancer treatments are intended to induce apoptosis through an extrinsic path or through a mitochondrial-intrinsic path via modulation of glucose metabolism [24]. Tumor cells show a surprisingly different metabolism compared to normal cells. The most important alteration is the Warburg effect $[25,26]$, which describes how tumor cells, even in the presence of abundant oxygen, continue to convert glucose to lactate with decreased mitochondrial respiration. Another important issue in cancer treatment is that hematologic cells strongly interact with the surrounding microenvironment, with bone marrow (BM) stroma potentially exerting protective effects from cytotoxic actions of drugs. In our present study, we screened the antitumor activity of ruxolitinib and vorinostat, each alone and in combination, in cell lines of hematologic malignancies including Hodgkin's disease and selected subtypes of non-Hodgkin's lymphoma, myeloma multiple, and chronic lymphatic leukemia. Our data suggested that all selected hematological malignancies were sensitive to ruxolitinib and vorinostat monotherapy, but the combination of drugs synergistically increased the inhibitory effects, leading the tumor cells to undergo cessation of growth, differentiation inhibition, and increased apoptosis. We further cocultured these 12 cell lines with mesenchymal cells, to examine how combined drug treatment competed with the protective effect exerted by the stroma. Finally, we examined metabolic aspects specifically the cellular depletion of ATP and lactate levels to better pinpoint a mechanism that could explain the effects of the drug combination. Our results suggested that the combination of ruxolitinib and vorinostat could affect proliferation by acting on the glycolytic and oxidative pathways. Since treatment with a combination of ruxolitinib and vorinostat showed a broad spectrum of action compared to individual drugs, such combined therapy may represent a promising new therapeutic modality for hematologic neoplasms.

\section{RESULTS}

\section{Ruxolitinib interacts with vorinostat to induce cytotoxic effects in treated cell lines}

First, we evaluated the cytotoxic effects $\left(\mathrm{IC}_{50}\right)$ in the 12 hematological cell lines after 24 and $48 \mathrm{~h}$ of treatment with ruxolitinib and vorinostat as single agents. We used the MTT assay to quantify the inhibition of cell viability. In all cell lines, the $\mathrm{IC}_{50}$ of ruxolitinib ranged from $12-20$ $\mu \mathrm{M}$ after $24 \mathrm{~h}$ of incubation, and from 1-5 $\mu \mathrm{M}$ after $48 \mathrm{~h}$. The $\mathrm{IC}_{50}$ of vorinostat in all cell lines ranged from 15-25 $\mu \mathrm{M}$ after $24 \mathrm{~h}$, and from $5-10 \mu \mathrm{M}$ after $48 \mathrm{~h}$.

To determine how viability was impacted by combined exposure to both ruxolitinib and vorinostat, we next incubated all cell lines for 24 and $48 \mathrm{~h}$ with both drugs at different concentration ratios, as indicated in the Chou-Talalay method, and again analyzed cell viability by MTT assay. We observed an additive effect $(\mathrm{CI}=1)$ in the WSU-NHL, Granta 519, Jeko1, U266, HUT78, and L540 cell lines after $24 \mathrm{~h}$ of simultaneous exposure to ruxolitinib and vorinostat. In the remaining cell lines (RL, RPMI8266, Karpas422, Karpas299, MEC1, and L1236) the drug combination induced a synergistic effect $(\mathrm{CI}<1)$ after $24 \mathrm{~h}$. More specifically, the $\mathrm{CI}$ values ranged from $0.1-0.3$, indicating strong synergy according to the Chou-Talalay theory (Figure 1). Notably, treatment with a combination of the two drugs at low concentrations for 48 $\mathrm{h}$ was too cytotoxic, causing obvious cell death revealed by exclusion assay with $0.2 \%$ Trypan Blue.

\section{Combination of ruxolitinib and vorinostat exerts a synergistic cytotoxic effect on co-cultures of mesenchymal stem cell and tumor cell lines}

Many studies support the theory that the bone marrow micro-environment can confer growth benefits and induce drug resistance in malignant cells $[27,28$, 29]. Since we observed that different cell lines varied in their responses to the combination of ruxolitinib and vorinostat, we used a co-culture assay to investigate the possible protective effect of $\mathrm{BM}$ mesenchymal stem cells on the 12 cell lines. Ruxolitinib and vorinostat, alone and in combination, showed very weak cytotoxic effects (2-5\%) after $24 \mathrm{~h}$ on all 12 cell lines in co-culture with hMSCs. This indicated defensive action of the BM microenvironment after $24 \mathrm{~h}$ of treatment. After $48 \mathrm{~h}$, ruxolitinib-related cytotoxicity increased to $15-20 \%$ in all cell lines co-cultured with hMSCs, while treatment with vorinostat alone for $48 \mathrm{~h}$ induced a moderate decrease of cell viability (3-5\%) in all 12 cell lines. After $48 \mathrm{~h}$ of co-culturing with ruxolitinib plus vorinostat, all cell lines showed an increase in cell death (45-55\%), particularly the six more sensitive cell lines. After $72 \mathrm{~h}$ of co-culturing with both drugs, the six most sensitive cell lines did 
not show an increased percentage of viable cells, while the other cell lines continued to be protected by the microenvironment (Figure 2). The six more sensitive cell lines were cultured for $120 \mathrm{~h}$, and the viability assay confirmed the prolonged synergistic effect of combined treatment with both drugs over the protective function of mesenchymal cells. Ruxolitinib and vorinostat, alone and in combination, exerted no cytotoxic effects on stromal cells alone from 24 to $120 \mathrm{~h}$.
Ruxolitinib and vorinostat, alone and in combination, regulate apoptosis via caspase activation and regulating anti-apoptotic proteins

To determine the apoptotic effects of ruxolitinib and vorinostat, alone and in combination, on all 12 cell lines, we evaluated the fraction of annexin V-positive cells (early and late apoptosis). After $24 \mathrm{~h}$ of single-drug treatment with ruxolitinib $(5 \mu \mathrm{M})$ and vorinostat $(10 \mu \mathrm{M})$, the
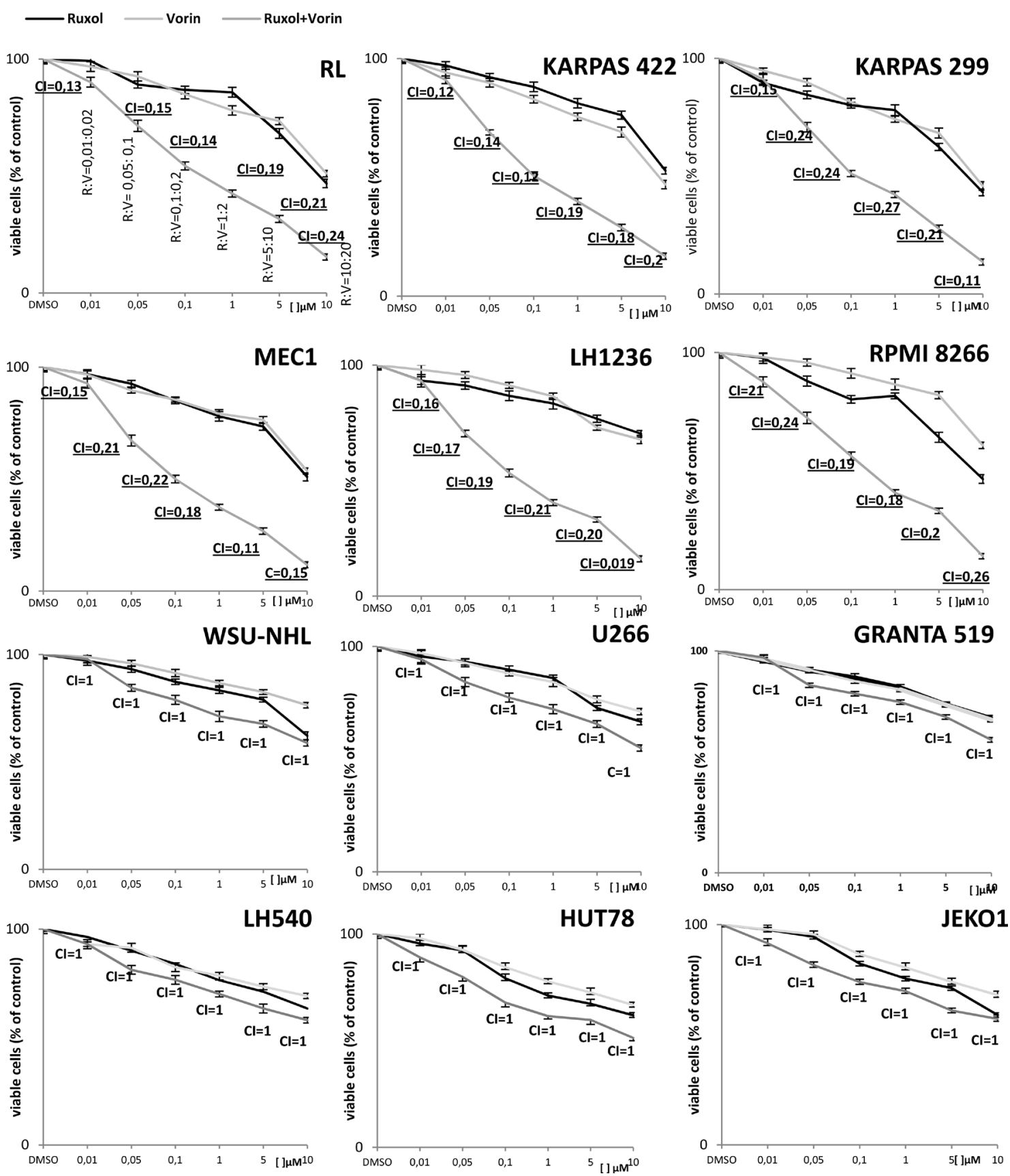

Figure 1: The antiproliferative activity of ruxolitinib and vorinostat, alone and in combination, after $24 \mathrm{~h}$ of treatment in all 12 cell lines. Cell viability was evaluated by MTT assay. Results represent the mean \pm standard error obtained from three independent experiments. To determine whether the combination of ruxolitinib with vorinostat was additive or synergistic, we investigated fixed ratios of combination of the drugs ( $\mathrm{R}: \mathrm{V}=1: 2)$, and applied isobologram analysis based on the Chou-Talalay method. Combination indices (CI) are reported in each graph. 
percentage of apoptotic cells was not more than 5-10\%. On the other hand, combined treatment with these drugs for $24 \mathrm{~h}$ led to an increase of the apoptotic fraction to $40-50 \%$ (Figure 3 ). Additionally, treatment of cells with ruxolitinib and vorinostat, alone and in combination, led to activation of caspase cleavage. Compared to single-agent treatment, combined treatment triggered substantially greater caspase- 3 and caspase- 8 activation in all 12 cell lines. Caspase- 9 cleavage was detected only in the six more sensitive cell lines. To confirm whether ruxolitinib plus vorinostat caused caspase cascade, we treated all cell lines with the pan-caspase inhibitor z-VAD-fmk $(10 \mu \mathrm{M})$ prior to combined drug treatment for $24 \mathrm{hrs}$. As shown in Figure 4, z-VAD-fmk remarkably restrained the cell apoptosis induced by ruxolitinib plus vorinostat in the sensitive cell line LH1236 and in the less sensitive cell line LH540. Comparable results were obtained in the other cell lines (data not shown).

To better clarify the apoptosis mechanism induced by ruxolitinib and vorinostat, we evaluated the expressions of some apoptosis-regulating proteins that induce programmed cell death (BAX, BID, and BAD), and of apoptosis inhibitors (BCL-2 and MCL-1). Expression levels were measured as mean fluorescence intensity (MFI) by flow cytometry after $24 \mathrm{~h}$ of incubation. In all cell lines, treatments with both single drugs and with the drug combination were associated with increased expressions of the BAX, BID, and BAD pro-apoptotic proteins. With regards to the anti-apoptotic proteins
BCL-2 and MCL-1, the combination of ruxolitinib with vorinostat was associated with downregulation of both proteins in the six most sensitive cell lines, while singledrug treatment did not have this effect (Table 1).

\section{The combination of ruxolitinib and vorinostat influences the cell cycle and related proteins}

In all 12 cell lines, ruxolitinib and vorinostat treatment alone did not significantly affect the cell cycle distribution. On the other hand, the combination treatment induced cell cycle progression defects, with a marked increase of G2-M arrest after $24 \mathrm{~h}$. Compared to single-agent treatment, cells subjected to combined treatment with ruxolitinib and vorinostat showed a slight decrease of cells in S phase and sub G0/G1 phase, and a significantly decreased percentage of cells in $\mathrm{G} 0$ / G1 (Figure 5A and 5B). To investigate the effects of ruxolitinib and vorinostat on cell cycle-related proteins, we evaluated the expressions of AURORA A, CCND1, p27, and p21 in all 12 cell lines. Combination drug treatment induced upregulated expression of the CDK inhibitors p21 and p27, and downregulated expression of the positive regulators of cell cycle progression AURORA A and CCND1 (Figure 5C-5E). The results of densitometric semi-quantification of western blot bands were normalized by total proteins to the untreated control levels of p27, p21, AURORA A, and CCND1. Figure 5 presents the effects of combined treatments in the RL cell

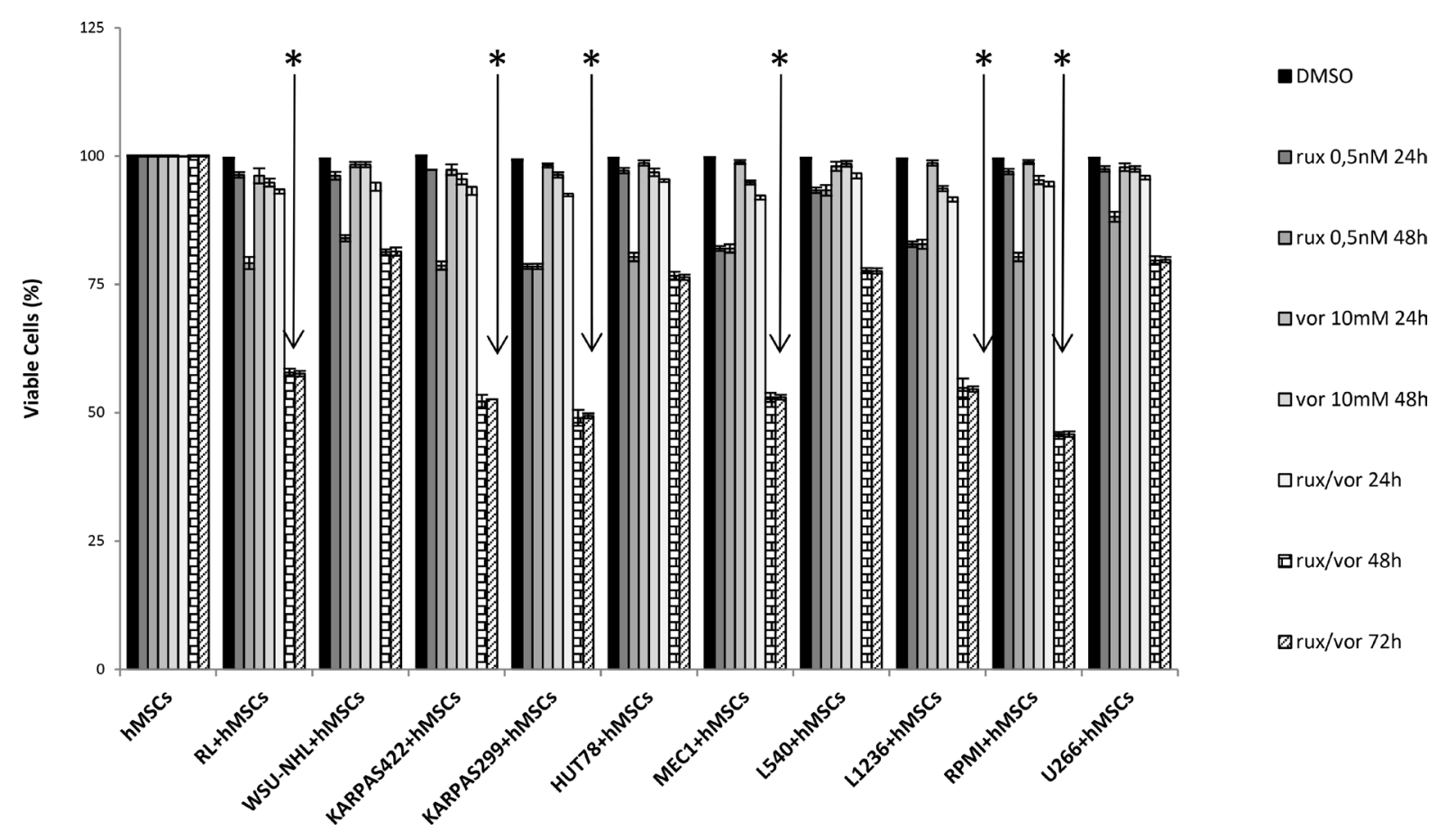

Figure 2: Viability of 12 cell lines in vitro when co-cultured with the stromal cell line hMSC for 24 to 72 h. Tumor cells were harvested and stained with Trypan blue to determine cellular viability. Graphs indicate the viability index used to normalize the viability values to those under control conditions. Values are the mean \pm standard error of three experiments. ${ }^{*} P<0.001$ Statistically significant differences versus control and single agents. 
Table 1: Expressions of apoptosis-regulating proteins that induce programmed cell death (BAX, BID, and BAD) and of apoptosis inhibitors (BCL-2 and MCL-1)

\begin{tabular}{|c|c|c|c|c|c|c|c|c|c|c|c|c|c|}
\hline & & RL & WSU & K422 & HUT78 & K299 & MEC-1 & L-540 & L-1236 & RPMI & U266 & JEKO1 & GRANTA \\
\hline & & \multicolumn{12}{|c|}{$\mathrm{N}$-fold increase/decrease in median MFI } \\
\hline & & \multicolumn{12}{|c|}{ (N-fold-proportion of protein expression (MFI) in examined sample vs untreated control, ns.-not significant) } \\
\hline \multirow[t]{3}{*}{ BAX } & $\mathrm{R}$ & 2,06 & 1,82 & 1,45 & 2 & 1,49 & 2,06 & 1,64 & 1,98 & 1,89 & 1,75 & 1,32 & 1,69 \\
\hline & $\mathrm{V}$ & 2,11 & 1,8 & 1,54 & 1,6 & 1,63 & 2,13 & 1,601 & 1,92 & 1,85 & 1,7 & 1,28 & 1,72 \\
\hline & $\mathrm{R}+\mathrm{V}$ & 2,13 & 1,91 & 1,65 & 2,1 & 1,8 & 2,14 & 1,7 & 2,1 & 2,04 & 1,87 & 1,47 & 1,79 \\
\hline \multirow[t]{3}{*}{ BID } & $\mathrm{R}$ & 1,89 & 1,71 & 0,84 & 1,84 & 2,13 & 2,04 & 1,42 & 2,1 & 2,001 & 1,86 & 1,6 & 1,79 \\
\hline & $\mathrm{V}$ & 1,92 & 1,72 & 0,87 & 1,82 & 2,06 & 1,85 & 1,56 & 2 & 1,94 & 1,81 & 1,56 & 1,72 \\
\hline & $\mathrm{R}+\mathrm{V}$ & 1,94 & 1,84 & 1,09 & 1,87 & 2,4 & 2,32 & 1,63 & 1,9 & 2,05 & 2,1 & 1,64 & 1,89 \\
\hline \multirow[t]{3}{*}{ BAD } & $\mathrm{R}$ & 1,69 & 1,52 & 1,35 & 1,89 & 1,52 & 1,63 & 1,64 & 1,36 & 2,05 & 1,91 & 1,2 & 1,32 \\
\hline & V & 1,43 & 1,41 & 1,46 & 1,96 & 1,41 & 1,69 & 1,59 & 1,29 & 2,09 & 1,98 & 1,26 & 1,27 \\
\hline & $\mathrm{R}+\mathrm{V}$ & 1,64 & 1,49 & 1,35 & 2,13 & 1,74 & 1,98 & 1,84 & $2,1^{*}$ & 2,04 & 2,03 & 1,52 & 1,63 \\
\hline \multirow[t]{3}{*}{ BCL2 } & $\mathrm{R}$ & 0,91 & 0,35 & 1,2 & 0,89 & 0,91 & 2,3 & 1,56 & 1,54 & 2,1 & 0,38 & 1,06 & 0,95 \\
\hline & V & 0,85 & 0,41 & 1,05 & 1,01 & 1,2 & 2,07 & 1,39 & 1,62 & 1,89 & 0,41 & 0,97 & 0,9 \\
\hline & $\mathrm{R}+\mathrm{V}$ & $0,26^{*}$ & 0,24 & $0,65^{*}$ & 0,65 & $0,35^{*}$ & $0,36^{*}$ & $0,76^{*}$ & $0,37^{*}$ & $0,51^{*}$ & 0,32 & 0,98 & 0,74 \\
\hline \multirow[t]{4}{*}{ MCL-1 } & $\mathrm{R}$ & 1,93 & 0,63 & 2,1 & 0,69 & 2,23 & 1,32 & & 0,95 & 1,89 & 1,58 & 1,15 & 0,84 \\
\hline & $\mathrm{V}$ & 1,72 & 0,59 & 2,13 & 0,99 & 2 & 1,09 & 0,95 & 1,04 & 2,14 & 1,46 & 0,98 & 0,53 \\
\hline & $\mathrm{R}+\mathrm{V}$ & $0,84^{*}$ & 0,59 & $0,48^{*}$ & 0,54 & $0,61^{*}$ & $0,37^{*}$ & 0,74 & $0,29^{*}$ & $0,54^{*}$ & $0,67^{*}$ & 0,78 & 0,55 \\
\hline & \multicolumn{13}{|c|}{ *statistically significant differences vs control } \\
\hline
\end{tabular}

Expression levels were measured as mean fluorescence intensity (MFI) by flow cytometry after $24 \mathrm{~h}$ of incubation with ruxolitinib plus vorinostat. Data are the mean of three different experiments. ${ }^{*} P<0.001$ Statistically significant differences versus control and single agents.

line. This cell line was randomly chosen for display, and the results obtained in the RL cell line are comparable with the other 11 cell lines.

\section{Combination of ruxolitinib and vorinostat enhances dephosphorylation of proteins of the JAK-STAT pathways}

In all 12 cell lines, we tested the ability of ruxolitinib and vorinostat, alone and in combination, to inhibit some JAK family members. Without treatment, all cell lines expressed comparable levels of total JAK2, p-JAK2, total STAT3, p-STAT3, total STAT5, and p-STAT5 proteins (Figure 6A). Following $24 \mathrm{~h}$ of treatment with ruxolitinib $(5 \mu \mathrm{M})$, we found diminished p-JAK2 expression in the RL, RPMI8266, and Karpas299 cell lines. All cell lines showed diminished expressions of the p-STAT3 and p-STAT5 proteins, as can be observed from the densitometric analysis reported in Figure 6B. Vorinostat treatment alone did not significantly alter p-JAK2 expression in any cell line, and slightly impacted the expressions of p-STAT3 and p-STAT5 in all cell lines (Figure 6C). Finally, $24 \mathrm{~h}$ of treatment with the combination of ruxolitinib and vorinostat $($ ratio $=1: 2)$ caused marked suppressive effects on the expressions of p-JAK2, p-STAT3, and p-STAT5 in all cell lines (Figure 6D).

\section{Ruxolitinib and vorinostat influence secretion of IL-10, IL-6, and IL-17A}

JAK-STAT pathways are activated through tyrosine phosphorylation of the cytoplasmic domains of cytokine receptors upon cytokine binding. In particular, IL-6, IL10, and IL-17A signaling occurs through both JAK1 and JAK2 [30-32]. We evaluated the expressions of IL-6, IL-10, and IL-17A in all 12 cell lines relative to control. After $24 \mathrm{~h}$ of treatment with the combination of ruxolitinib and vorinostat, all 12 cell lines showed significantly decreased $(P<0.001)$ secretion of IL-10 into the media (Figure 7A). Moreover, all 12 cell lines showed similar decreases in IL-6 secretion after treatment with ruxolitinib and vorinostat, both alone and in combination (Figure 7B). Interestingly, combination drug treatment particularly inhibited sustained production of IL-17A in the six more sensitive cell lines (Figure 7C).

\section{Ruxolitinib and vorinostat affect autophagy}

Autophagy plays a complex and often contradictory role in tumor progression, and is a promising target for cancer treatment [33]. To study autophagy, we quantified the p62 protein response in all 12 cell lines following treatment with ruxolitinib and vorinostat, alone and in combination. Quantitative determination of $\mathrm{p} 62$ revealed 


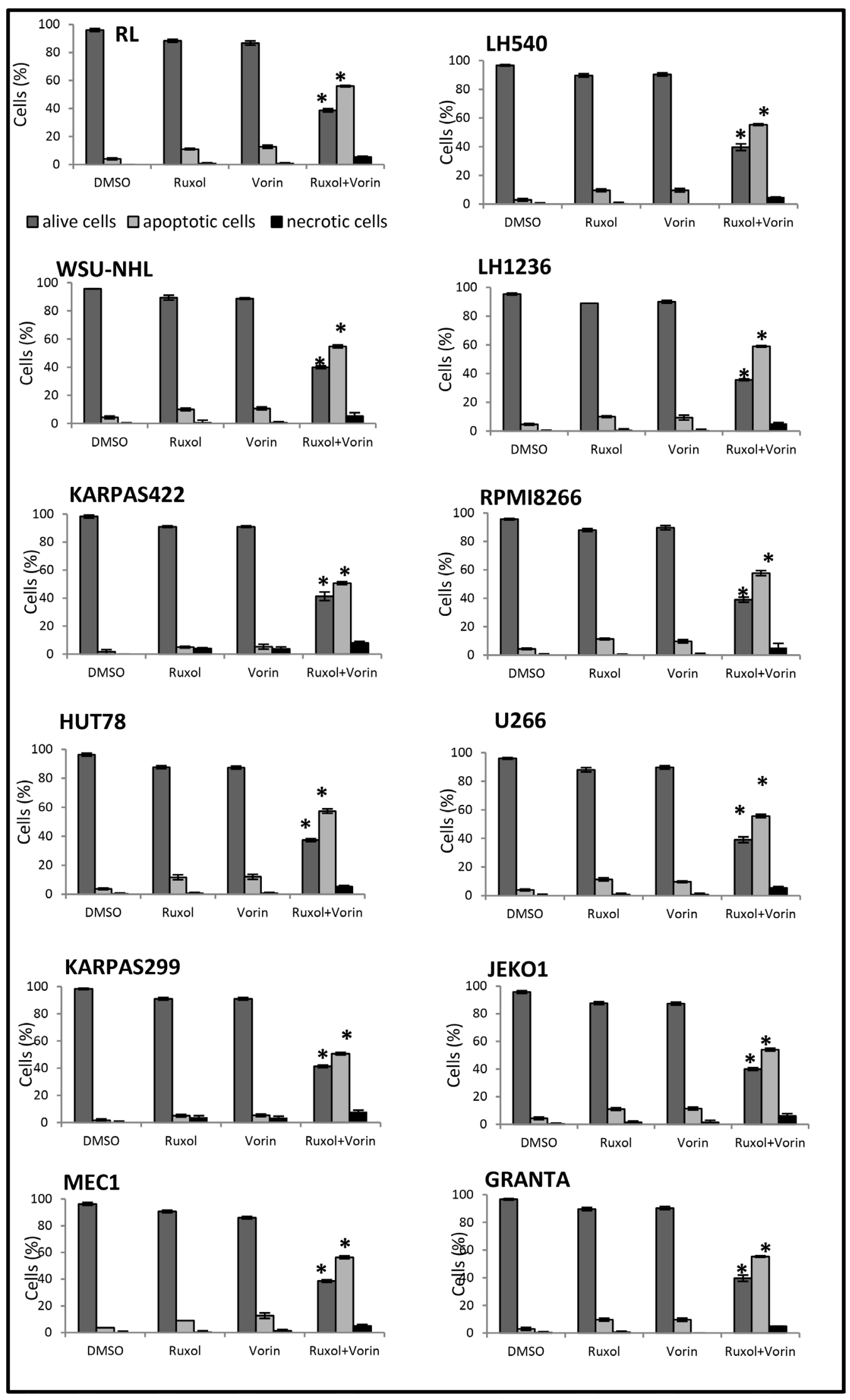

Figure 3: Pro-apoptotic effects of ruxolitinib and vorinostat, alone and in combination. Flow cytometric analysis revealed increased apoptosis after $24 \mathrm{~h}$ of combined treatment. ${ }^{*} P<0.001$ versus single-drug treatment. 
that treatment with a combination of ruxolitinib and vorinostat induced autophagy comparable to that observed in cells subjected to $12 \mathrm{~h}$ of serum starvation. Combined treatment with the two drugs along with the autophagy inhibitor chloroquine inhibited autophagic flux of cancer (Figure 8). In the same cellular lysate used to study autophagy, we also evaluated cellular apoptosis (data not shown). The results suggest that the combination of ruxolitinib and vorinostat induced both apoptosis and autophagy.

\section{Exposure to the ruxolitinib and vorinostat combination triggers ROS generation}

We previously found that the combination of ruxolitinib and vorinostat triggers the mitochondriamediated signaling pathway. Alterations in the redox state of apoptotic cells could be related to activation of the final stage of the caspase cascade $[34,35]$. ROS generation could play an important role in cytotoxicity induced by ruxolitinib and vorinostat. Thus, we investigated the effects of these drugs, both alone and in combination, with co-administration of N-acetyl cysteine (NAC) antioxidants to block ROS generation. Single-agent administration of ruxolitinib or vorinostat exerted only a slight-to-moderate effect on ROS generation in all 12 cell lines (Figure 9). Combined drug treatment resulted in a moderate increase of ROS generation in the WSU and U266 cell lines-about 1.5-fold higher than the untreated controls. Moreover, combined drug treatment induced a significant $(P<0.001)$ increase of ROS production in the six more sensitive cell lines. Co-treatment with NAC effectively blocked ROS generation in all 12 cell lines.

\section{Combined treatment with ruxolitinib and vorinostat decreased ATP generation, lactate levels and GLUT1 expression}

Highly proliferative cells, such as cancer cells, are characterized by alterations in energy metabolism, including increased anaerobic glycolysis [36]. We initially measured the amount of ATP in culture medium after cell lines were incubated for $24 \mathrm{~h}$ with ruxolitinib (5 $\mu \mathrm{M})$ and vorinostat $(10 \mu \mathrm{M})$, alone and in combination.
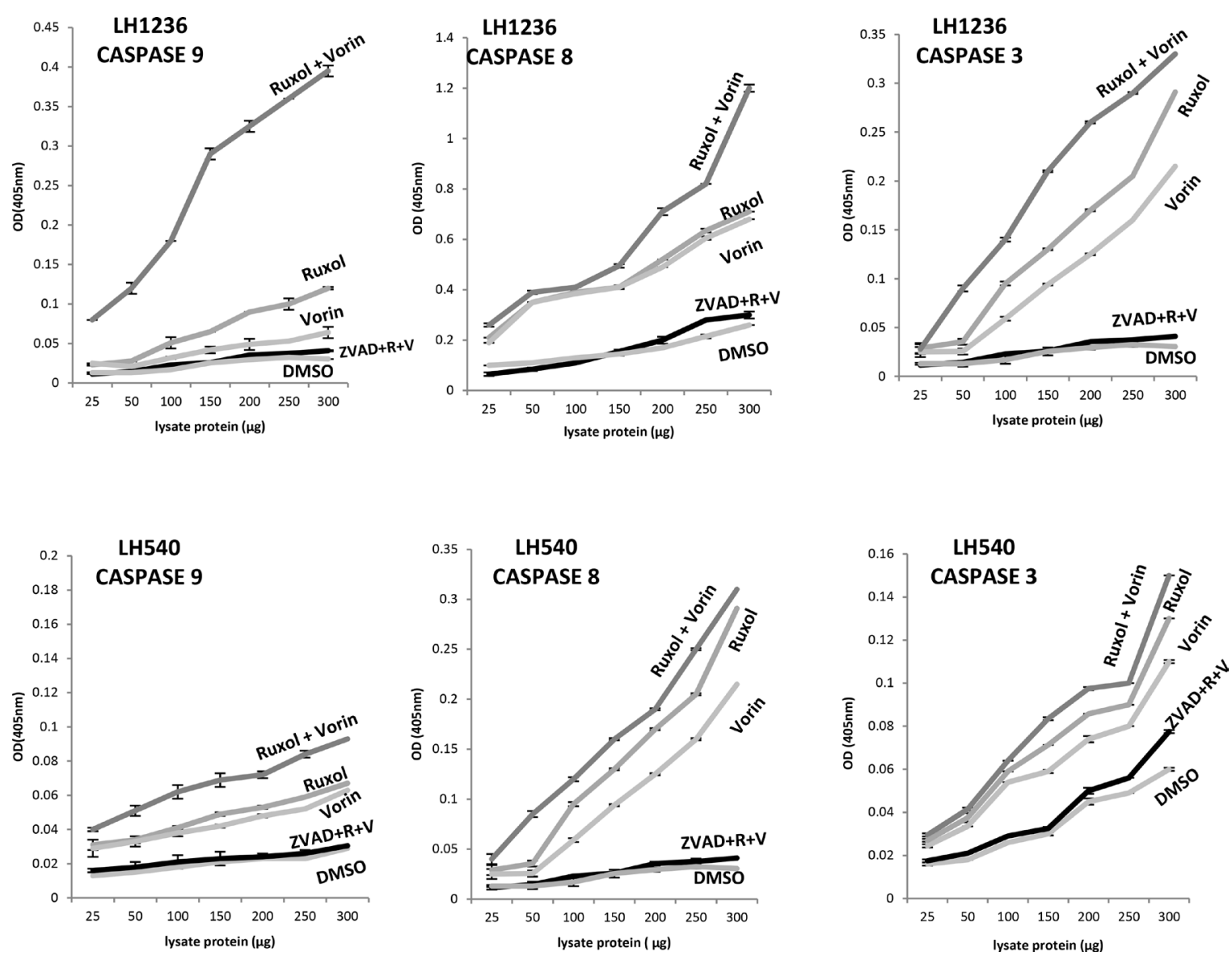

Figure 4: Caspase activation triggered in 12 cell lines by exposure to ruxolitinib ( $5 \mu \mathrm{M})$ and vorinostat (10 $\boldsymbol{\mu M})$, alone and in combination (ratio of 1:2). Caspase-8, caspase-9, and caspase 3 protease assays were used to assess the caspase proteolytic activity in lysates of cells following treatment with ruxolitinib and vorinostat, alone and in combination. The graph shows absorbance data obtained from treated RL cell lines. Co-exposure of cells to ruxolitinib and vorinostat led to markedly increased caspase activity in the six more sensitive cell lines. 
We observed a decreased glycolytic rate in the six more sensitive cell lines following treatment with ruxolitinib plus vorinostat (Figure 10). Addition of the mitochondrial ATP synthase inhibitor oligomycin led to an increased glycolytic rate in both treated and untreated cell lines. We also monitored the amount of lactate released in all 12 cell lines. The six more sensitive cell lines showed decreased lactate production after incubation with ruxolitinib combined with vorinostat (Figure 11). Lactate production can vary in accordance with any alteration of the glycol pathway; however, our experiments were performed in an in vitro model with cultured cells, thus limiting fluctuations in lactate levels. Based on our results, we further examined whether cells treated with the combined drugs would show decreased expression of GLUT1, the key enzyme of the glycolytic pathway. After $24 \mathrm{~h}$ of incubation with ruxolitinib plus vorinostat, we detected decreased GLUT1 expression in all 12 cell lines, with no significant difference between the most sensitive cell lines and the remaining cell lines (Figure 12).

\section{DISCUSSION}

JAK-2 dysregulation plays an important role as an oncogenic driver, prompting increasing interest in JAK-2 inhibitors for therapy against hematological malignancies
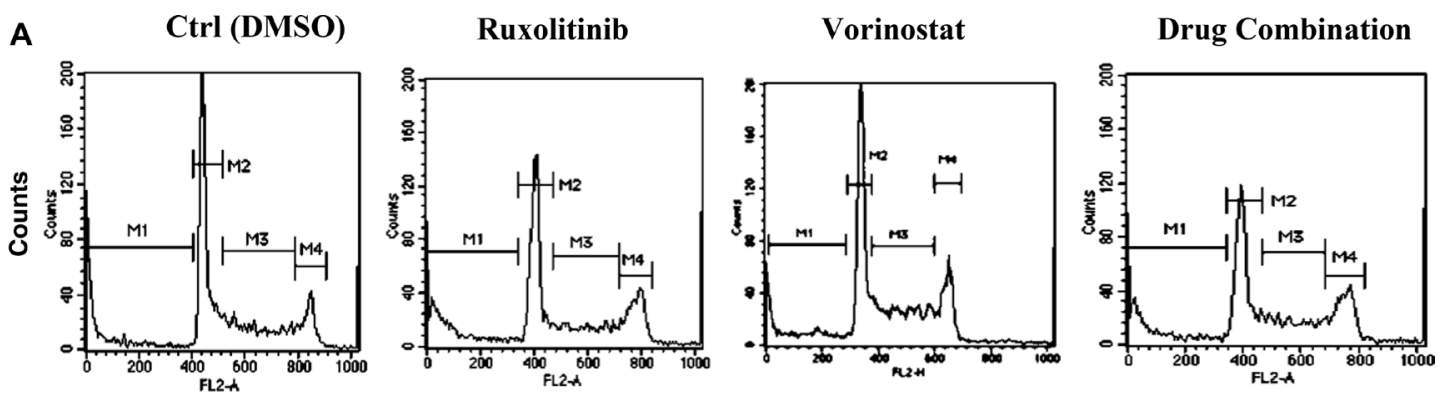

PI - staining of DNA FL2-H
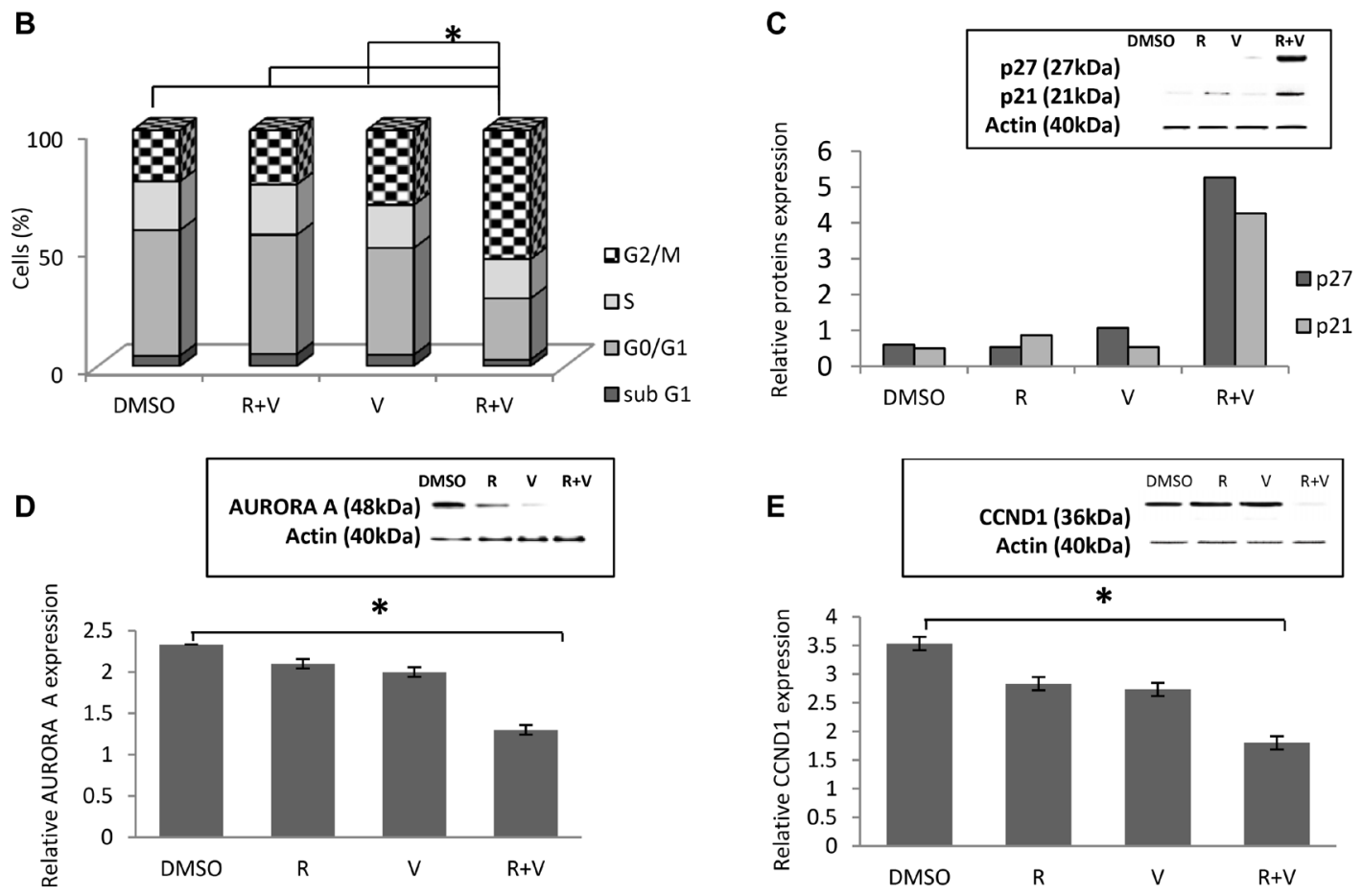

Figure 5: Treatment with ruxolitinib plus vorinostat influences the cell cycle and related proteins. (A) Representative flow cytometry histograms of the cell cycle distribution of the RL cell line. M1 indicates sub-G0/g1; M2, G0/G1; M3, S; and M4, G2/M. (B) Cell cycle profile of RL cells treated for $24 \mathrm{~h}$ with ruxolitinib $(5 \mu \mathrm{M})$ and vorinostat $(10 \mu \mathrm{M})$, alone and in combination (ratio, 1:2). Bars represents the mean \pm standard error estimated based on the rate of cells in the following cell cycle fraction: sub-G1, G0-G1, S, and G2-M. ${ }^{*} p<0.001$ Statistically significant differences versus control and single agents. (C-E) Western blot of cellular extracts from RL cell line. The levels of p21, p27, AURORA A, and CCND1 were analyzed using Quantiti One software (Bio-Rad Laboratories) and expressed as relative value compared to total proteins. ${ }^{*} p<0.001$ Statistically significant differences versus control and single agents. 
$[37,38]$. The translation of first-generation JAK inhibitors (tofacinib, oclacitinib, baricitinib, and ruxolitinib) to clinical application in myelofibrosis represents a substantial advance in patient treatment (ClinicalTrials. gov, U.S. National Institutes of Health, Bethesda, MD, USA: http://clinicaltrials.gov/). The success of ruxolitinib has inspired the rapid development of additional JAK inhibitors, extending the armamentarium for targeted therapy of malignancies driven by JAK signaling [39].
However, the curative potential of JAK inhibitors appears to be limited, and the survival benefits are controversial with limited follow-up available [40]. Since JAK-2 plays an essential role hematopoiesis, JAK-2 inhibition is associated with hematologic toxicities that limit dose escalation, which may in turn limit the extent of target inhibition. HDAC inhibitors reduce JAK-2 expression, likely due to effects on JAK-2 mRNA expression and through increased JAK-2 proteasomal deterioration
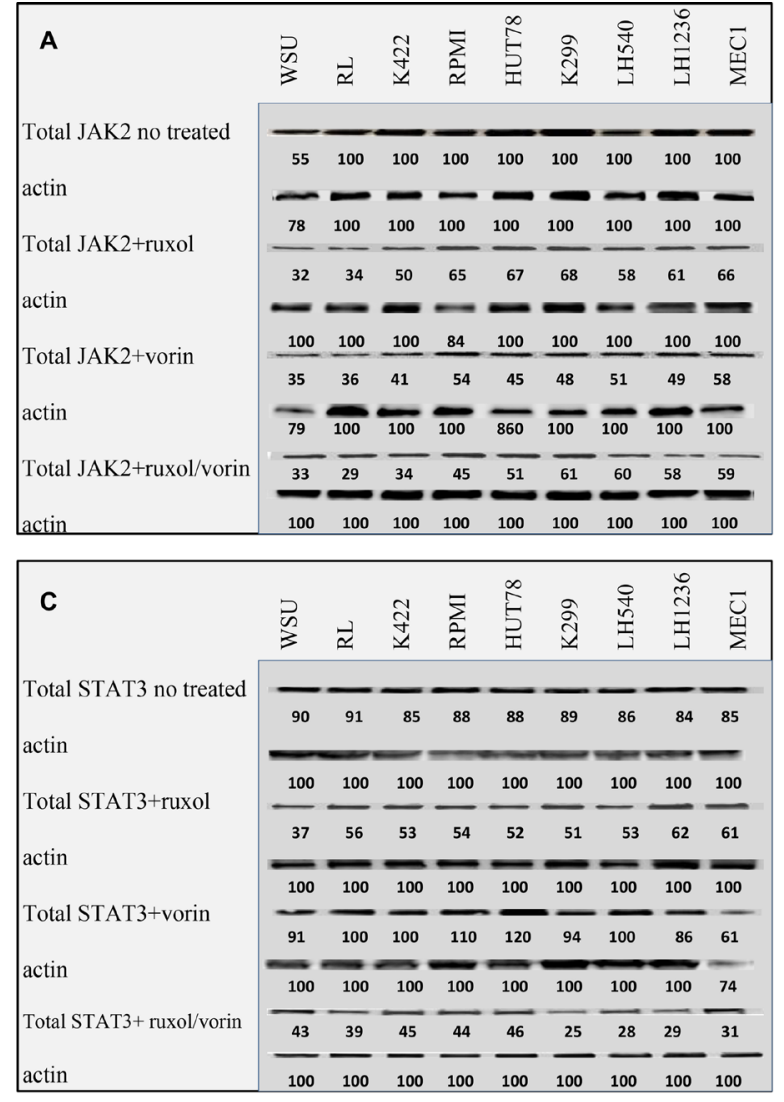
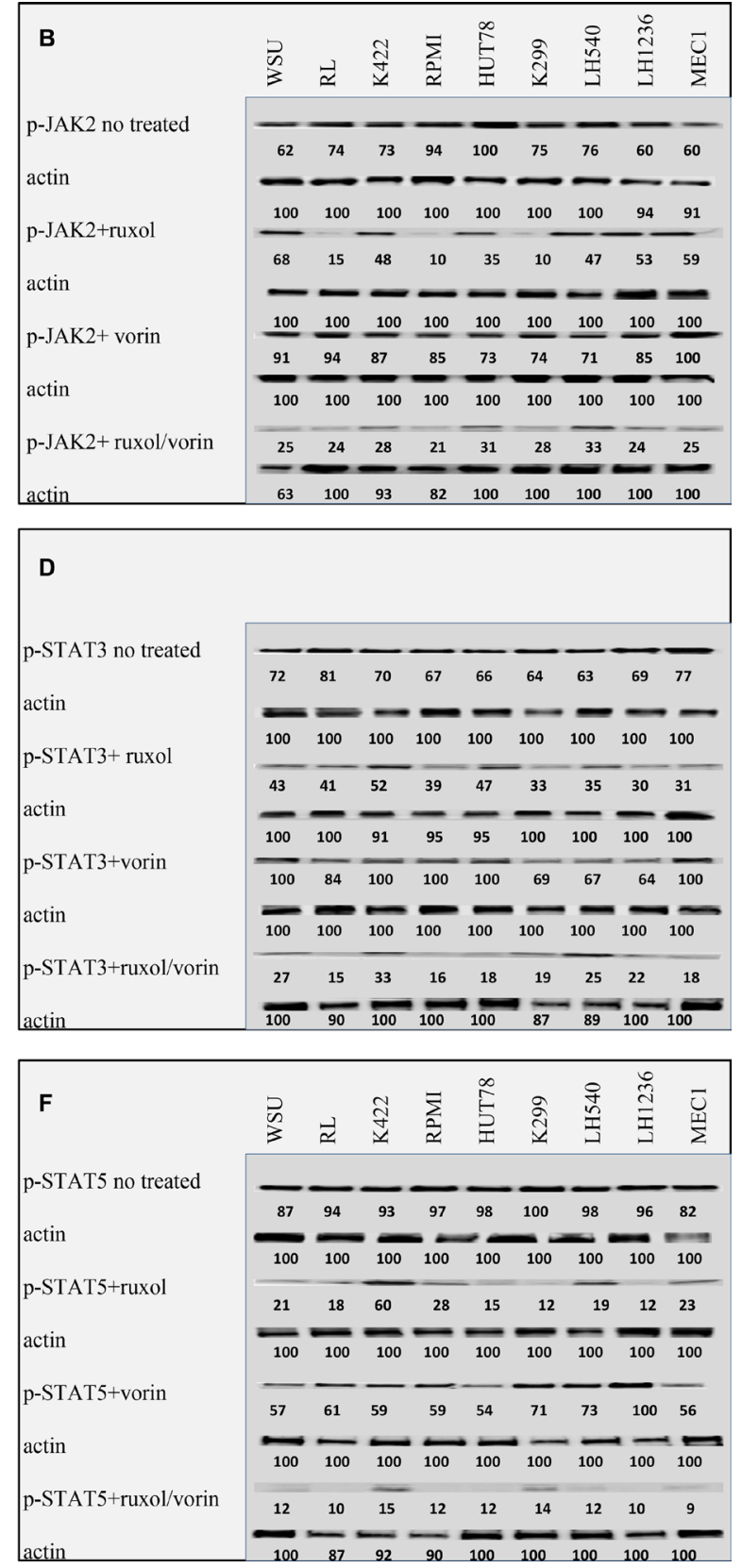

Figure 6: Western blot analysis of JAK2-STAT3-5 pathway protein levels in cell lines after $24 \mathrm{~h}$ of treatment with ruxolitinib $(5 \mu \mathrm{M})$ and vorinostat $(10 \mu \mathrm{M})$, alone and in combination (ratio, 1:2). Whole-cell lysates from cell lines no treated, treated with Ruxolitinib, with Vorinostat and with the combination were subjected to protein gel blotting using the indicated antibodies (A-F). Densitometric semi-quantification of bands normalized to the untreated control is shown below the immunoblot bands. The samples come from the same experiment and that the gels / blots have been treated in parallel. In the cell lines not shown in the figure we obtained blots comparable. 
[41-43]. Combination therapy might prove beneficial due to synergistic impacts on oncogenic transformation, could enable the effective use of lower doses of the different agents with better tolerability, and might avoid or delay the development of drug resistance.

Our present results show that combination treatment with ruxolitinib and vorinostat had improved effects on proliferation and apoptosis in an in vitro model of hematological disease. We found that a combination of the two drugs showed greater efficacy compared to lowdose single-agent treatment with ruxolitinib and vorinostat in 12 cell lines, including two B-cell lymphomas, two T lymphoma, one chronic B-cell leukemia, two myeloma multiple, two Hodgkin lymphoma, two mantle lymphoma, one non-Hodgkin lymphoma. The results of combination treatment were additive in both mantle cell lymphoma cell lines, in one LH cell line, in one myeloma cell line, in one T-cell lymphoma cell line, and in one B-cell lymphoma cell line. In the remaining cell lines, the combination of the two drugs had highly synergistic effects. These results led us to postulate that this combination may have the potential to overcome protective effects of a tumor microenvironment. Indeed, tumor cells treated with the combination of ruxolitinib and vorinostat for $48 \mathrm{~h}$ were not protected by coculture with stromal cells. The synergistic cytotoxic action of this drug combination was maintained for up to $120 \mathrm{~h}$. We also found that the ruxolitinib and vorinostat combination influenced the cell cycle distribution, increasing G2-M

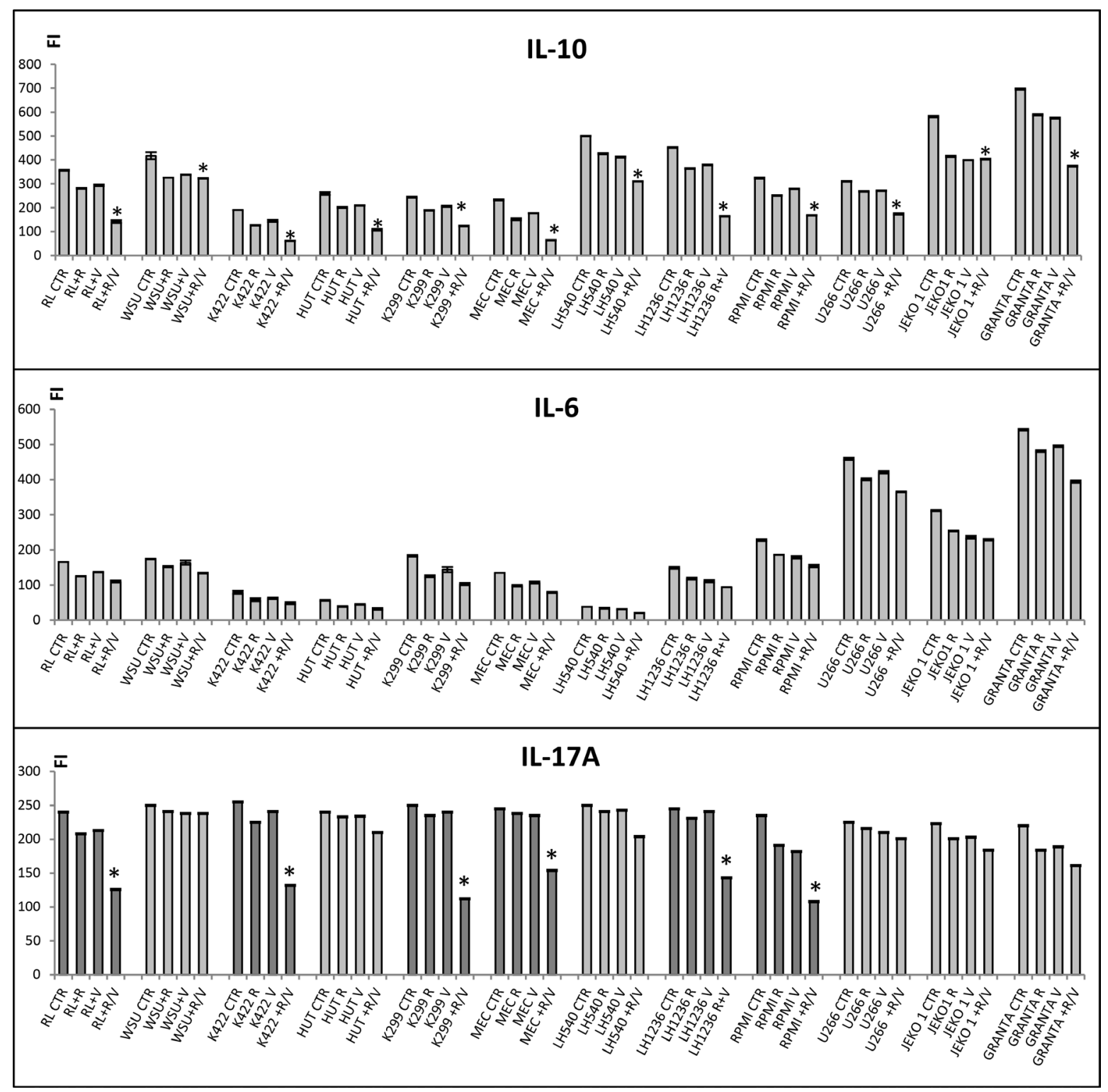

Figure 7: Secretion of IL-10, IL-6, and IL-17A after treatment with ruxolitinib and vorinostat. Cells were stimulated with ruxolitinib $(5 \mu \mathrm{M})$ and vorinostat $(10 \mu \mathrm{M})$, alone and in combination (ratio, 1:2), and a multiplex assay was used to determine the levels of IL-10, IL-6, and IL-17A secreted into the media. In all graphs, error bars represent the standard error of three independent experiments. ${ }^{*} P<0.001$ versus control and single agents. 
phase arrest after $24 \mathrm{~h}$ in all 12 cell lines. Correspondingly, the combined treatment affected the related cell cycle proteins. In all 12 cell lines, treatment with ruxolitinib and vorinostat for $24 \mathrm{~h}$ resulted in upregulation of the cell cycle regulators $\mathrm{p} 21$ and $\mathrm{p} 27$, and downregulation of CCND1 and AURORA A, which are important in regulating the cell cycle control points. These results confirmed that this combination of drugs induced tumor growth inhibition.

After $24 \mathrm{~h}$ of single-agent treatment with ruxolitinib or vorinostat, less than $10 \%$ of cells were apoptotic annexin

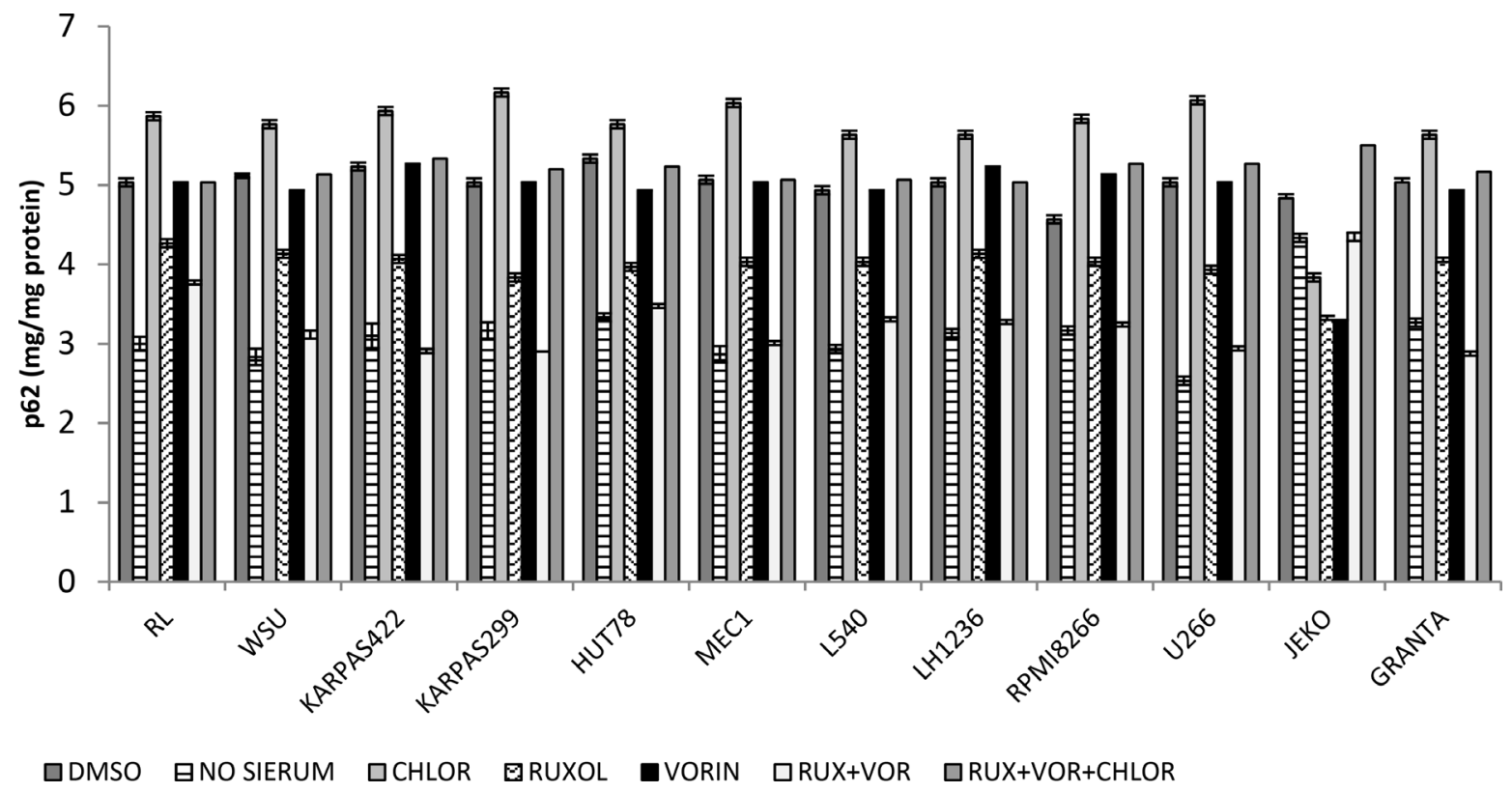

Figure 8: Quantitative determination of $\mathrm{p}-62$ expression in all 12 cell lines after treatment for $24 \mathrm{~h}$ with ruxolitinib $(5 \mu \mathrm{M})$ and vorinostat $(10 \mu \mathrm{M})$, alone and in combination. Results represent the mean \pm standard error of three independent experiments.

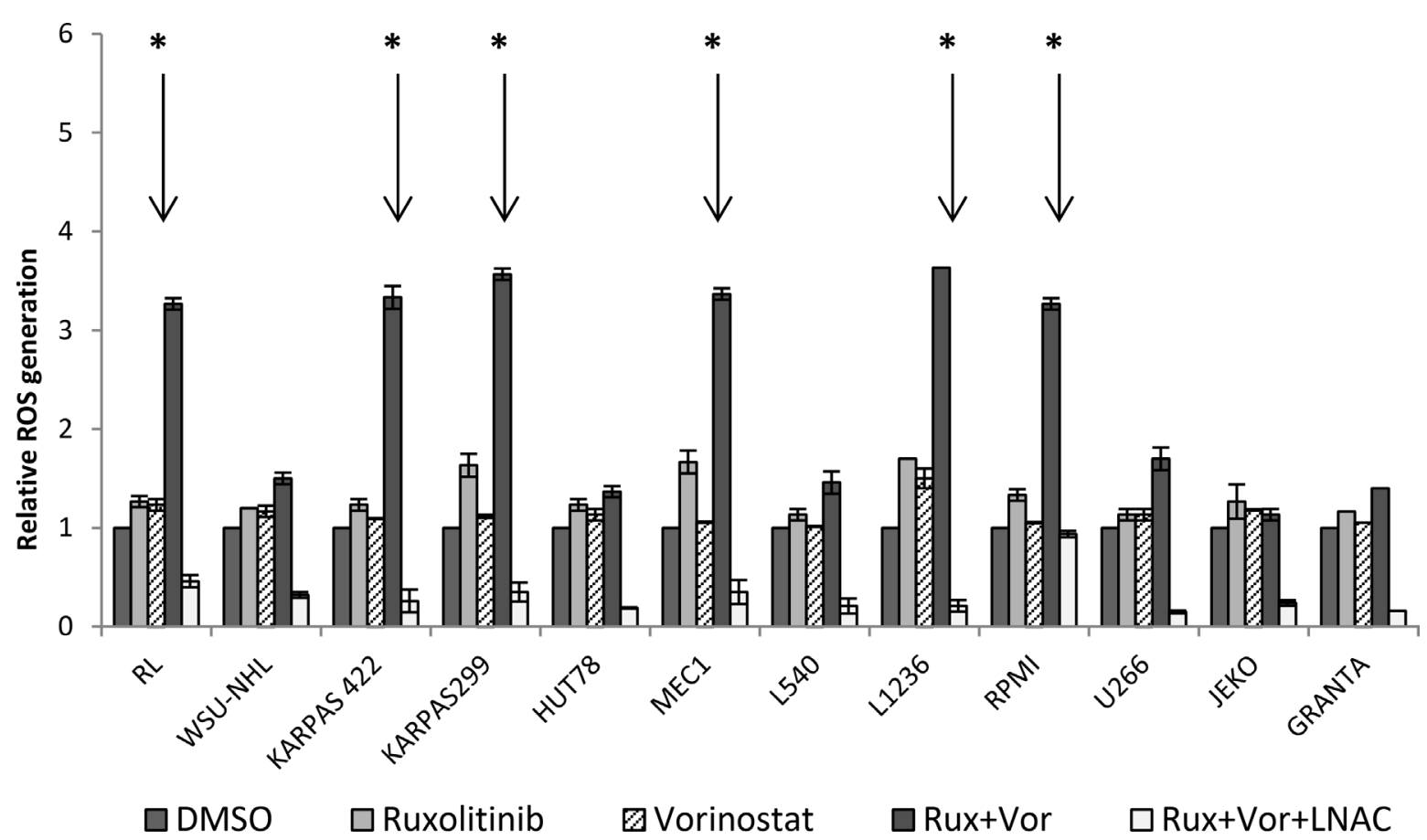

Figure 9: Treatment with ruxolitinib and vorinostat, alone and in combination, affects ROS generation. ROS levels were determined by flow cytometry, and histograms show quantitative analysis of ROS generation. Increased ROS levels were observed in the six more sensitive cell lines after combined drug treatment for $24 \mathrm{~h}$. Co-administration of the antioxidant $\mathrm{N}$-acetyl cysteine NAC blocked the increase of ROS generation. Results represent the mean \pm standard error obtained from three independent experiments. ${ }^{~} P<0.001$. 
V-positive cells. However, this proportion of apoptotic cells increased to $50 \%$ in all 12 cell lines after treatment with ruxolitinib combined with vorinostat. Caspases play a very important role in programmed cell death and are thus attractive targets for the development of new cancer therapies. We chose to study the caspase initiator caspase-8, and the caspase effectors caspase- 9 and caspase- 3 . After 24 of combined drug treatment, we detected cleavage of caspase- 8 and caspase- 3 in all 12 cell lines, suggesting activation of the extrinsic apoptotic pathway. Moreover, only the six most sensitive cell lines showed increases in the cleaved form of caspase-9, suggesting caspase activation of mitochondrial events regulating intrinsic apoptosis. The most sensitive cell lines also differed from the other cell lines in terms of the regulation of anti-apoptotic proteins. After treatment with the drug combination, all 12 cell lines showed increased expression of the pro-apoptotic proteins BAX, BID and BAD, but only the six more sensitive cell lines showed downregulation of the apoptotic inhibitors BCL-2 and MCL-1. These results confirmed the possibility that ruxolitinib combined with vorinostat may differentially target signaling pathways in the six more sensitive cell lines.
JAK/STAT activation has been demonstrated in hematological malignancies, providing the rationale for various therapeutic approaches involving JAK kinase inhibitors [44-46]. Here we assessed JAK/STAT activation based on the expressions of phospho-JAK2, STAT3, and STAT5. Notably, the combination of ruxolitinib and vorinostat increased the de-phosphorylation of JAK2, STAT3, and STAT5, blocking the pathway. This may be partly due to ruxolitinib's inhibitory effect on JAK2, but may also be influenced by the fact that vorinostat targets the JAK pathway. It has been reported that when cells become resistant to JAK2 inhibitors, they remain dependent on the expression of JAK2 proteins; thus, our present findings may suggest alternative therapy that indirectly degrades JAK2 [47]. JAK/STAT activation is closely related to binding of cytokines to their receptors. Tumors contain high numbers of circulating cytokines, making them attractive as potential therapeutic targets. Binding of IL-6, IL-10, and IL-17 to their receptors promotes the activation of JAKs [48, 49]; however, the anti-tumor immunomodulatory role of IL-6, IL-10 and IL-17 remains unclear and appears contradictory in some aspects. All three interleukins are released into healthy

\section{Metabolic study in B cell lymphoma cell lines}

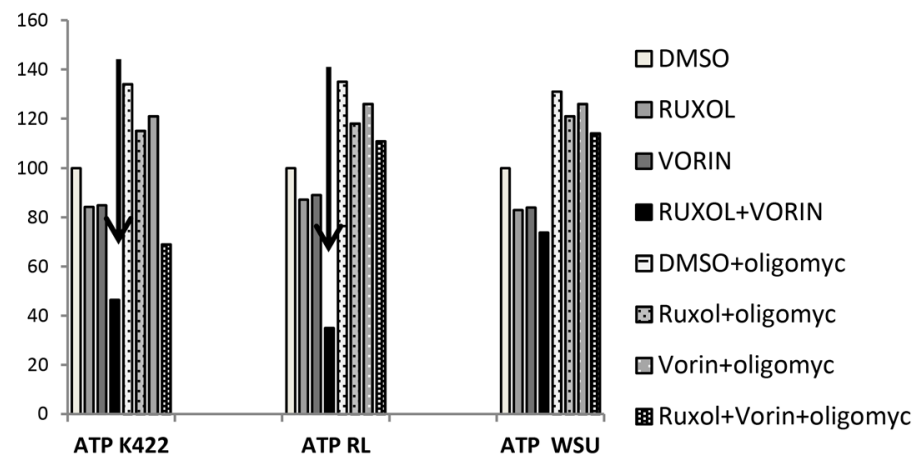

Metabolic study in T cell lymphoma cell lines

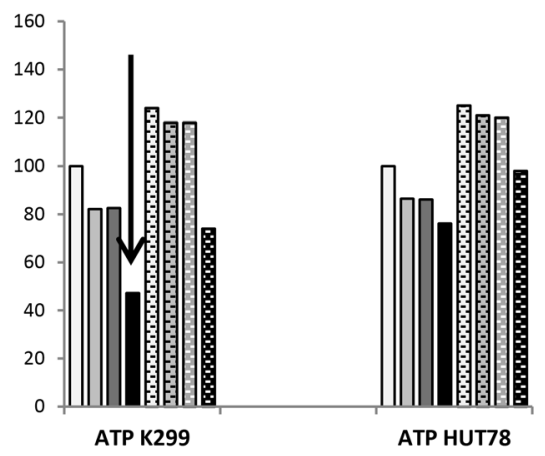

Metabolic study in $\mathrm{H}$ lymphoma and chronic B cell leukemia
Metabolic study in mantle cell lymphoma
Metabolic study in mieloma multiple
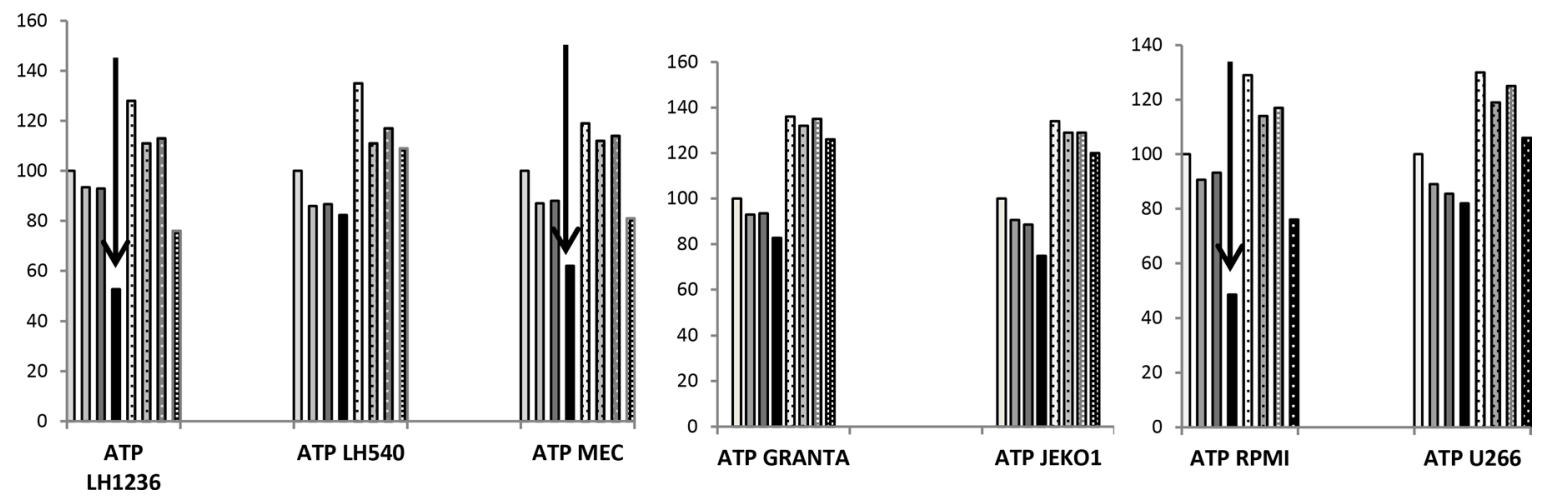

Figure 10: Intracellular ATP levels in response to ruxolitinib and vorinostat treatment. Intracellular ATP levels were measured in control and drug-treated cells after $24 \mathrm{~h}$. ATP levels are expressed as $\%$ of untreated control. Data represent mean \pm standard error of three independent experiments. 
tissues, as well as into many tumor infiltrates in larger quantities. The ability of interleukins to provide access to tissues and to recruit large numbers of immune cells has obvious advantages for antitumor responses, especially in solid tumors. However, cytokines that facilitate immune cell access to cancers may also generate conditions that facilitate tumor cell migration and diffusion via circulation [50]. Our present experiments showed that ruxolitinib combined with vorinostat reduced IL-10 and IL-6 secretion comparably among all 12 cell lines. On the other hand, IL-17A expression was only significantly reduced after combined treatment in the six more sensitive cell lines. These results suggest that the drug combination had a broad range of effects that acted upstream of JAK/STAT pathway inhibition.

JAK is reportedly involved in autophagy induction for immune regulation in various cancer cells [51-52]. Thus, we investigated the cross-talk between the JAK

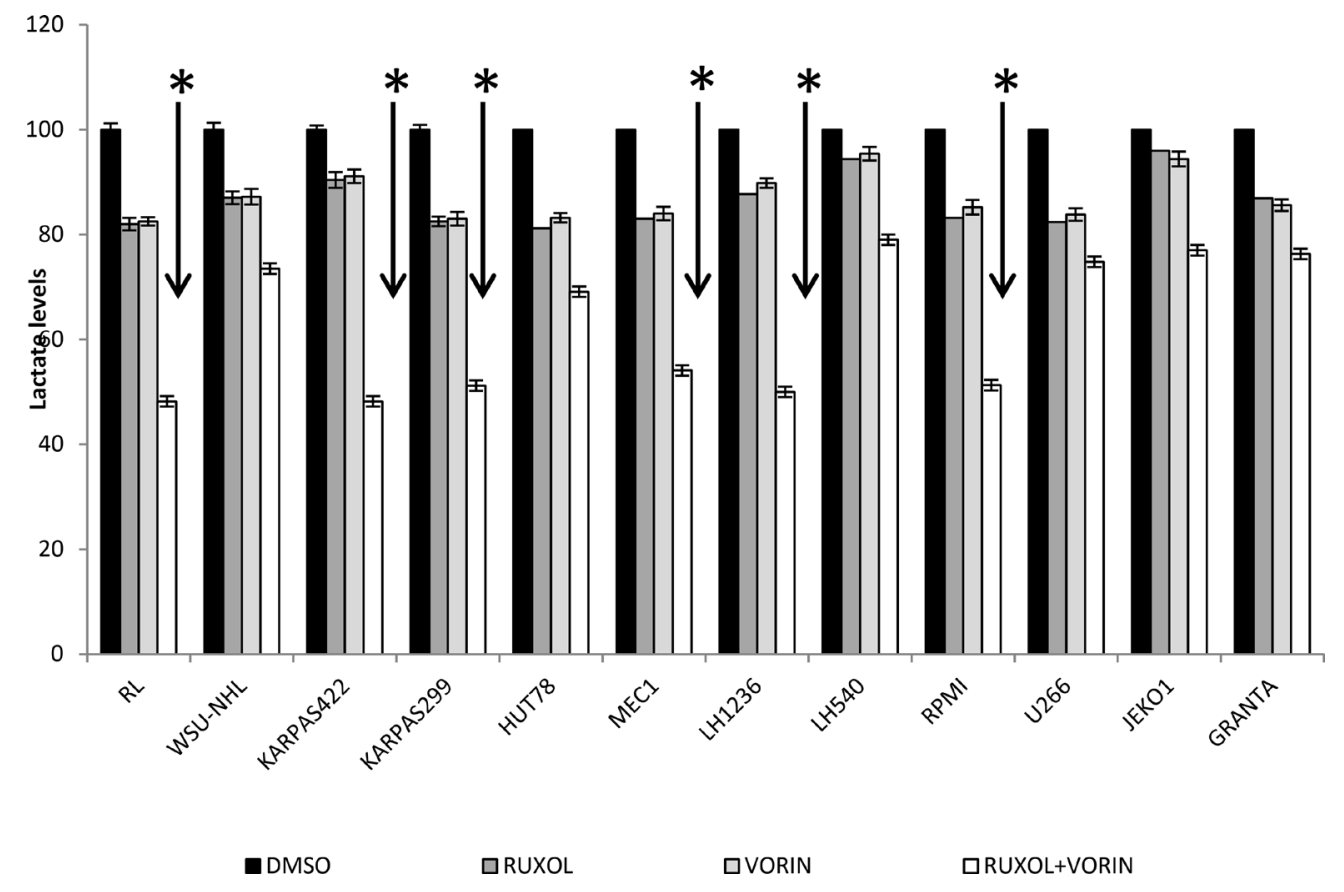

Figure 11: Lactate levels after treatment with ruxolitinib $(5 \mu M)$ and vorinostat $(10 \mu M)$, alone and in combination. Cells were cultures with drugs (alone and in combination) for $24 \mathrm{~h}$. Then the supernatant was collected and lactate levels were determined by colorimetric analysis. Data represent mean \pm standard deviation of three independent experiments. ${ }^{*} p<0.001$ Statistically significant differences versus control and single agents.

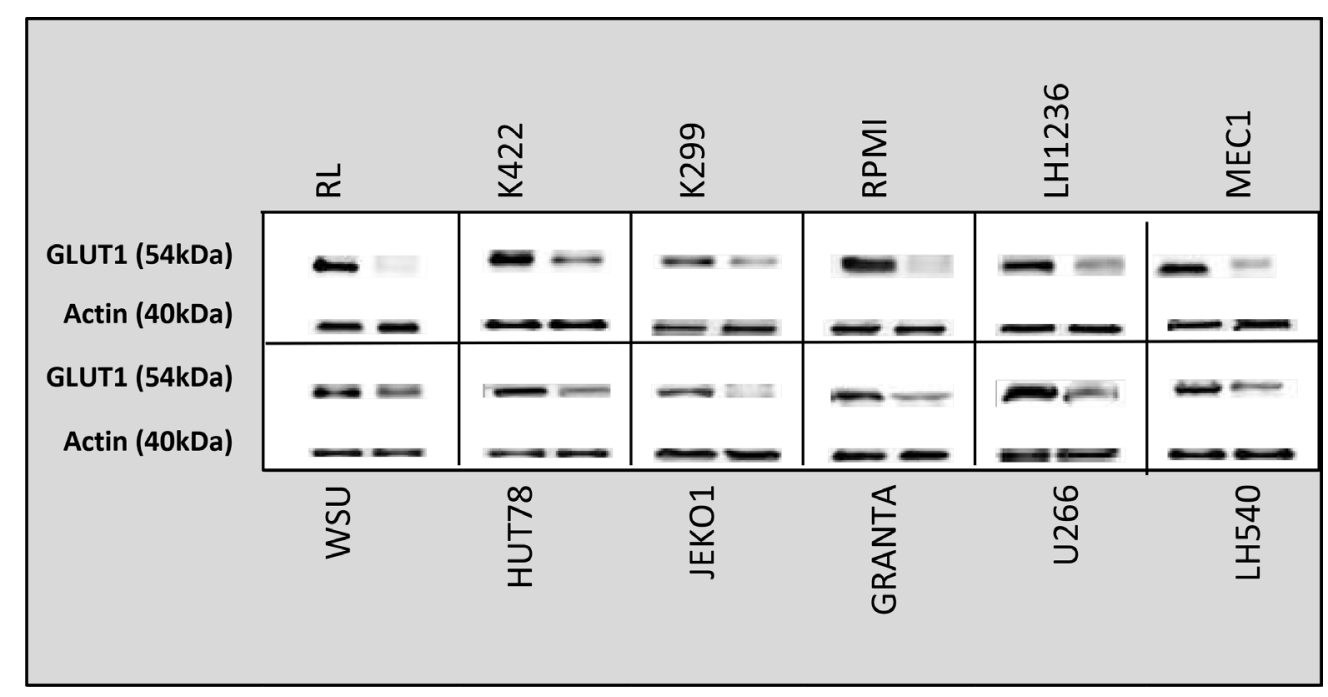

Figure 12: GLUT1 protein levels in all 12 cell lines after treatment with ruxolitinib plus vorinostat for $24 \mathrm{~h}$. Densitometric semi-quantification of bands normalized to the untreated control is shown below the immunoblot bands. 
pathway and the autophagy process in our treated cell lines. We showed that ruxolitinib and vorinostat, alone and in combination, induced a decrease of the amount of the autophagy-related p62 protein in cancer cells, indicating activation of the autophagic system. Autophagy and apoptosis are mechanisms that cause increased oxidative stress, leading to ROS production. Increased ROS production is detected in various cancers, and reportedly plays several roles linked to pro-tumorigenic signaling, as well as roles related to anti-tumorigenic signaling, initiating oxidative stress-induced tumor cell death [55]. Our present results indicated that ruxolitinib and vorinostat synergistically induced apoptosis in the six more sensitive cell lines, associated with markedly increased ROS generation. Co-administration of the ROS scavenger LNAC reduced the increased ROS levels induced by the ruxolitinib and vorinostat combination. These results could be very interesting considering that tumor cells have an altered redox balance compared to their normal counterparts, which identifies ROS manipulation as a potential target for cancer therapies. Cancer cells exhibit increased rates of aerobic glycolysis, and the modulation of glucose metabolism reportedly affects intrinsic and extrinsic apoptosis. Our data show that combined treatment with ruxolitinib plus vorinostat altered tumor cell metabolism by reducing the glycolic state, lead to the greater sensitivity of the six cell lines.

In conclusion, here we show that treatment with a combination of ruxolitinib and vorinostat led to decreased glucose metabolism, and that this partial reversion of the Warburg effect was associated with ROS production, apoptosis, and cell growth inhibition. Testing these drugs under different cell culture conditions provided information regarding drug efficacy, and could help predict pharmacological effects that may be difficult to translate from in vitro and ex vivo conditions to clinical practice. Our present results offer evidence of synergistic interaction between ruxolitinib and vorinostat in hematological tumor cells, and provide the rationale to support clinical studies using the combination of both agents in patients.

\section{MATERIALS AND METHODS}

\section{Cell lines}

We purchased the mantle cell lymphoma cell lines GRANTA519 and Jeko1, multiple myeloma cell lines U266 and RPMI8266, B-cell lymphoma cell lines Karpas422 and RL, cutaneous T-cell lymphoma cell line HUT78, anaplastic cell lymphoma cell line Karpas 299, chronic B-cell leukemia cell line MEC1, and Hodgkin lymphoma cell lines LH540 and LH1236 from Deutsche Sammlung von Mikroorganismen und Zellkulturen GmbH. Cell line characteristics are available online (http://www. dsmz.de/human_and_animal_cell_lines/main). The B-cell lymphoma cell line WSU-NHL was kindly provided by
Dr. M. Introna (Bergamo, Italia). GRANTA519 and MEC1 cells were cultured in Iscove's MEM supplemented with $10 \%$ fetal bovine serum (FBS), $2 \mathrm{mM}$ L-glutamine, penicillin $(100 \mathrm{U} / \mathrm{mL})$, and streptomycin $(100 \mathrm{U} / \mathrm{mL})$. All other cell lines were cultured in RPMI-1640 supplemented with $10 \%$ fetal bovine serum (FBS), 2 mM L-glutamine, penicillin $(100 \mathrm{U} / \mathrm{mL})$, and streptomycin $(100 \mathrm{U} / \mathrm{mL})$ (all purchased from Euroclone). The human mesenchymal stem cells (hMSC) were purchased from Tebu Bio and cultured in complete MSC expansion media (Tebu Bio). Cells in the logarithmic growth phase were used for experiments.

\section{Drugs}

Ruxolinostat and vorinostat were purchased from Selleck Chemicals. Each was dissolved in dimethylsulfoxide (DMSO; Euroclone) to create $10^{-2} \mathrm{M}$ stock solutions that were stored at $-80^{\circ} \mathrm{C}$. These stock solutions were further diluted using cell culture medium to the appropriate concentrations for use. Oligomycin was purchased from Sigma Aldrich.

\section{Assessment of cell viability and proliferation}

In dose-response experiments, cell lines were treated with increasing concentrations of ruxolitinib and vorinostat for 24 and $48 \mathrm{~h}$. Relative viable cells were determined by MTT assay (cellTiter non-radioactive cell proliferation assay; Promega). The absorbance at $550 \mathrm{~nm}$ was measured using an ELISA reader. We determined the drug concentration required for $50 \%$ inhibition of cell proliferation $\left(\mathrm{IC}_{50}\right)$ using Calcusyn software (Biosoft, Cambridge, UK) applying the median-effect method. Viability was assessed by exclusion assay with $0.2 \%$ Trypan Blue (Euroclone). DMSO was directly added to control samples and used as a drug solvent for drugtreated samples, and the DMSO concentration was kept constant $(0.1 \%)$ among treatments for all experiments. The maximum final concentration of DMSO $(<0.1 \%)$ did not affect cell proliferation and did not induce cytotoxicity in the tested cell lines.

\section{Combination study}

To investigate the inhibitory effects of drug combination treatment, we used isobologram analysis, following the Chou-Talalay method that provides algorithms for automated computer simulation of synergism and/or antagonism based on the median-effect equation derived from the mass action law $[53,54]$. We used CalcuSyn Windows software for dose-effect analysis and synergism/antagonism quantification (Biosoft, Cambridge, United Kingdom). The evaluation of drug synergism based on a median-effect equation is commonly described in the literature [55]. A combination index (CI) 
of $>1$ indicates antagonism, a CI of 1 denotes additivity, and a $\mathrm{CI}$ of $<1$ indicates synergism. More specifically, CI values ranging from $0.1-0.3$ are considered to indicate strong synergism, 0.3-0.7 synergism, and $0.7-0.85$ moderate synergism.

\section{Co-culture of cell lines with bone marrow stromal cells}

Human mesenchymal stem cells hMSC cells were cultured following the recommended protocol. Mesenchymal cells were seeded in triplicate in 96-well plates, and incubated for $48 \mathrm{~h}$ to reach confluence. Once the 12 cell lines adhered to the CM stroma, the co-cultures were treated with ruxolitinib and vorinostat, alone or in combination. After 24, 48, 72, 96, and $120 \mathrm{~h}$, the cells were harvested and assessed for viability.

\section{Cytometric analysis for apoptosis}

To determine the apoptosis rate after 24 and 48 hours of drug exposure, we performed flow cytometry using the Annexin V/Propidium Iodide Staining kit (Miltenyi Biotec, Germany), following the manufacturer's instructions. At least 20,000 events were acquired using a FACSCalibur cytometer (Becton Dickinson, San Jose, CA, USA), which were then analyzed using FlowJo Software (Tree Star, Ashland, OR, USA).

In preparation for intracellular marker staining, the cells were washed with 1\% v/v FBS-PBS (staining buffer), fixed in $4 \% \mathrm{w} / \mathrm{v}$ paraformaldehyde $\left(20 \mathrm{~min}\right.$ at $\left.4{ }^{\circ} \mathrm{C}\right)$, and permeabilized with $0.1 \%$ saponin in PBS. Then the cells were incubated for $1 \mathrm{~h}$ at $4^{\circ} \mathrm{C}$ with primary antibodies against p-BAD (Ser112), BID, BAX, MCL-1 and BCL2 (all from Cell Signaling). Finally, the cells were incubated with fluorophore-tagged secondary antibodies for $45 \mathrm{~min}$ at $4^{\circ} \mathrm{C}$, and the cells were analyzed using a FACSCalibur flow cytometer. The results were analyzed using the CellQuest program. Whenever possible, the immunophenotype results were expressed as the percentage of positive cells.

We measured caspase-3, caspase-8, and caspase 9 activity using colorimetric assay kits purchased from Enzo Life Sciences, in accordance with the manufacturer's instructions. Samples were analyzed using an ELISA reader.

\section{Cell cycle analysis}

For cell cycle distribution analysis, cells were harvested after $24 \mathrm{~h}$ of drug treatment. These cells were fixed in ethanol (95\%) containing RNase $(10 \mathrm{~g} / \mathrm{mL})$, stained for $15 \mathrm{~min}$ with propidium iodide (PI, $50 \mathrm{~m} /$ $\mathrm{mL}$ ), and then analyzed with a FACSCalibur cytometer. We calculated the percentages of cells in the subG1/G0 (dead cells), G1/G0, S, and G2/M phases of the cell cycle (determined in relation to DNA histogram analysis) using Modifit LT software (Verity Software House, Topshem, ME, USA).

\section{Autophagy detection}

We quantified autophagy in cell lysates using the quantitative immunometric detection method provided by the p62 (sequestosome 1) ELISA kit (Enzo Life Science, Farmingdale, NY, USA) following the manufacturer's instructions.

\section{Measurement of reactive oxygen species (ROS) production}

Reactive oxygen species (ROS) production was analyzed by incubating cells with 2',7'-dicholorodihydrofluorescein diacetate (DCFH-DA; Sigma Aldrich) in complete medium for 30 minutes at $37^{\circ} \mathrm{C}$. Fluorescence was quantified with a FACSCalibur cytometer (Becton Dickinson), and these data were analyzed using FlowJo Software (Tree Star).

\section{Lactate and ATP assay}

We measured lactate and ATP levels using an L-lactate assay colorimetric kit and an ATP assay colorimetric kit (Abcam), following the manufacturer's instructions. In the L-lactate assay kit, lactate is oxidized by lactate dehydrogenase to generate a product that interacts with a probe, producing a color $(\mathrm{OD}=450 \mathrm{~nm})$. The ATP assay kit is based on the phosphorylation of glycerol, generating a product that can be colorimetrically quantified $(\mathrm{OD}=570 \mathrm{~nm})$.

\section{Western blot analysis}

After drug treatments, $1 \times 10^{6}$ cells were pelleted and then lysed using the Mammalian Cell Extraction Kit (BioVision, Milpitas, CA, USA) following the manufacturer's instructions. Proteins (100 $\mu \mathrm{g} /$ lane $)$ were electrophoresed on 4-20\% (w/v) Miniprotean TGX Precast Gels (Bio-Rad, USA), and then transferred to nitrocellulose membranes (Bio-Rad Laboratories, Hercules, CA, USA). Membranes were immunoblotted using the following primary antibodies: total JAK2, p-JAK2, total STAT3, p-STAT3, total STAT5, p-STAT5, CCND1, AURORA A, p21, p27 and GLUT-1. Next, the membranes were incubated with species-specific horseradish peroxidase (HRP)-conjugated secondary antibody. All above-mentioned antibodies were purchased from Euroclone. Blots were developed using SuperSignal West Pico Chemiluminescent Substrate (Thermo Scientific, Rockford, IL, USA), and images were acquired using Chemidoc XRS+ and analyzed with Image Lab Software v.3.0 (Bio-Rad Laboratories). 


\section{Measurement of IL-6, IL-10, and IL-17a in cell culture supernatants}

Cells were cultured at a density of $5 \times 10^{5}$ cells/ $\mathrm{mL}$ for $24 \mathrm{~h}$ with ruxolitinib and vorinostat, alone and in combination. Then the supernatants were collected and analyzed for levels of the cytokines IL-6, IL-10, and IL-17a using a Human Magnetic Luminex Assay (R\&D Systems), following the manufacturer's instructions.

\section{Statistical analysis}

All experiments were independently repeated three times, with multiple replicates within each run. Data are expressed as mean \pm standard error. We analyzed statistical differences between control and drug-treated cells using one-way ANOVA, and $P$ values less than .05 were assigned significance. Data were analyzed using the Stata 8.2/SE package (StataCorp LP, College Station, TX, USA).

\section{Author contributions}

MC conceived and designed the study. MC, MC, and SP performed the experiments and analyzed the data. $\mathrm{MC}$ wrote the paper. SS supervised the experiments. All authors contributed equally, and read and approved the final manuscript.

\section{ACKNOWLEDGMENTS}

This work was supported by grants from the Associazione Mantovana per la Ricerca sul Cancro and from the Associazione Angela Serra.

\section{CONFLICTS OF INTEREST}

All the authors declare no conflicts of interest.

\section{REFERENCES}

1. Quintás-Cardama A, Verstovsek S. Molecular pathways: JAK/STAT pathway: mutations, inhibitors, and resistance. Clin Cancer Res. 2013; 19:1933-1940.

2. Neubauer H, Cumano A, Müller M, Wu H, Huffstadt $\mathrm{U}$, Pfeffer K. JAK2 deficiency defines an essential developmental checkpoint in definitive hematopoiesis. Cell. 1998; 3:397-409.

3. Levine RL, Gilliland DG. JAK-2 mutations and their relevance to myeloproliferative disease. Curr Opin Hematol. 2007; 14:43-7.

4. Meyer SC, Keller MD, Chiu S, Koppikar P, Guryanova OA, Rapaport F, Xu K, Manova K, Pankov D, O'Reilly RJ, Kleppe M, McKenney AS, Shih AH, et al. CHZ868, a Type II JAK2 Inhibitor, Reverses Type I JAK Inhibitor Persistence and Demonstrates Efficacy in Myeloproliferative Neoplasms. Cancer Cell. 2015; 28:15-28.

5. Mullally A, Ebert BL. Janus reveals another face: the biologic rationale for targeting Janus kinase 2 in lymphoma. J Clin Oncol. 2012; 30:4168-70.

6. Diaz T, Navarro A, Ferrer G, Gel B, Gaya A, Artells R, Bellosillo B, Garcia-Garcia M, Serrano S, Martínez A, Monzo M. Lestaurtinib inhibition of the JAK/STAT signaling pathway in Hodgkin lymphoma inhibits proliferation and induces apoptosis. PLoS One. 2011; 6:e18856.

7. Wade R, Rose M, Neilson AR, Stirk L, Rodriguez-Lopez R, Bowen D, Craig D, Woolacott N. Ruxolitinib for the treatment of myelofibrosis: a NICE single technology appraisal. Pharmacoeconomics. 2013; 31:841-52.

8. Furqan M, Mukhi N, Lee B, Liu D. Dysregulation of JAKSTAT pathway in hematological malignancies and JAK inhibitors for clinical application. Biomark Res. 2013; 1:5.

9. Wade R, Hodgson R, Biswas M, Harden M, Woolacott N. A Review of Ruxolitinib for the Treatment of Myelofibrosis: A Critique of the Evidence. Pharmacoeconomics. 2017; 35:203-213.

10. Tefferi A. Challenges facing JAK inhibitor therapy for myeloproliferative neoplasms. N Engl J Med. 2012; 366:844-6.

11. Roskoski R Jr. Janus kinase (JAK) inhibitors in the treatment of inflammatory and neoplastic diseases. Pharmacol Res. 2016; 111:784-803.

12. Dao KH, Solti MB, Maxson JE, Winton EF, Press RD, Druker BJ, Tyner JW. Significant clinical response to JAK1/2 inhibition in a patient with CSF3R-T618I-positive atypical chronic myeloid leukemia. Leuk Res Rep. 2014; 3:67-9.

13. Delgado-Martin C, Meyer LK, Huang BJ, Shimano KA, Zinter MS, Nguyen JV, Smith GA, Taunton J, Winter SS, Roderick JR, Kelliher MA, Horton TM, Wood BL, et al. JAK/STAT pathway inhibition overcomes IL7-induced glucocorticoid resistance in a subset of human T-cell acute lymphoblastic leukemias. Leukemia. 2017 [Epub ahead of print].

14. Thomas SJ, Snowden JA, Zeidler MP, Danson SJ. The role of JAK/STAT signaling in the pathogenesis, prognosis and treatment of solid tumours. Br J Cancer. 2015; 113:365-71.

15. Pardanani A. JAK2 inhibitors in myelofibrosis. Clin Adv Hematol Oncol. 2011; 9:856-8.

16. Mazzacurati L, Lambert QT, Pradhan A, Griner LN, Huszar D, Reuther GW. The PIM inhibitor AZD1208 synergizes with ruxolitinib to induce apoptosis of ruxolitinib sensitive and resistant JAK2-V617F-driven cells and inhibit colony formation of primary MPN cells. Oncotarget. 2015; 6:40141-57. https://doi.org/10.18632/oncotarget.5653.

17. Heider U, von Metzler I, Kaiser M, Rosche M, Sterz J, Rötzer S, Rademacher J, Jakob C, Fleissner C, Kuckelkorn U, Kloetzel PM, Sezer O. Synergistic interaction of the 
histone deacetylase inhibitor SAHA with the proteasome inhibitor bortezomib in mantle cell lymphoma. Eur $\mathrm{J}$ Haematol. 2008; 80:133-42.

18. Wozniak MB, Villuendas R, Bischoff JR, Aparicio CB, Martínez Leal JF, de La Cueva P, Rodriguez ME, Herreros B, Martin-Perez D, Longo MI, Herrera M, Piris MA, Ortiz-Romero PL. Vorinostat interferes with the signaling transduction pathway of T-cell receptor and synergizes with phosphoinositide-3 kinase inhibitors in cutaneous T-cell lymphoma. Haematologica. 2010; 95:613-21.

19. Benedetti R, Conte M, Altucci L. Targeting Histone Deacetylases in Diseases: Where Are We? Antioxid Redox Signal. 2015; 23:99-126.

20. Nebbioso A, Dell'Aversana C, Bugge A, Sarno R, Valente S, Rotili D, Manzo F, Teti D, Mandrup S, Ciana P, Maggi A, Mai A, Gronemeyer H, Altucci L. HDACs class II-selective inhibition alters nuclear receptor-dependent differentiation. J Mol Endocrinol. 2010; 45:219-28.

21. Grant S, Easley C, Kirkpatrick P. Vorinostat. Nat Rev Drug Discov. 2007; 6:21-2.

22. Lee JH, Choy ML, Marks PA. Mechanisms of resistance to histone deacetylase inhibitors. Adv Cancer Res. 2012; 116:39-86.

23. Cai J, Wei H, Hong KH, Wu X, Zong X, Cao M, Wang P, Li L, Sun C, Chen B, Zhou G, Chen J, Ji M. Discovery, bioactivity and docking simulation of Vorinostat analogues containing 1,2,4-oxadiazole moiety as potent histone deacetylase inhibitors and antitumor agents. Bioorg Med Chem. 2015; 23:3457-71.

24. Warburg O. On respiratory impairment in cancer cells. Science. 1956; 124:269-70.

25. Mushtaq M, Darekar S, Klein G, Kashuba E. Different Mechanisms of Regulation of the Warburg Effect in Lymphoblastoid and Burkitt Lymphoma Cells. PLoS One. 2015; 10:e136142.

26. Zub KA, Sousa MM, Sarno A, Sharma A, Demirovic A, Rao S, Young C, Aas PA, Ericsson I, Sundan A, Jensen ON, Slupphaug G. Modulation of cell metabolic pathways and oxidative stress signaling contribute to acquired melphalan resistance in multiple myeloma cells. PLoS One. 2015; 10:e0119857.

27. Weisberg E, Wright RD, McMillin DW, Mitsiades C, Ray A, Barrett R, Adamia S, Stone R, Galinsky I, Kung AL, Griffin JD. Stromal-mediated protection of tyrosine kinase inhibitor-treated BCR-ABL-expressing leukemia cells. Mol Cancer Ther. 2008; 7:1121-9.

28. Estrov Z, Quintás-Cardama A, Burger J, Zhang Y, Livun A, Knez L, Harris D, Creighton CJ, Kantarjian HM, Verstovsek $\mathrm{S}$, Manshouri T. Bone marrow stroma-secreted cytokines protect JAK2(V617F)-mutated cells from the effects of a JAK2 inhibitor. Cancer Res. 2011; 71:3831-40.

29. Striz I, Brabcova E, Kolesar L, Sekerkova A. Cytokine networking of innate immunity cells: a potential target of therapy. Clin Sci (Lond). 2014; 126:593-612.
30. Singh B, Schwartz JA, Sandrock C, Bellemore SM, Nikoopour E. Modulation of autoimmune diseases by interleukin (IL)-17 producing regulatory T helper (Th17) cells. Indian J Med Res. 2013; 138:591-4.

31. Roxburgh CS, McMillan DC. Therapeutics targeting innate immune/inflammatory responses through the interleukin-6/ JAK/STAT signal transduction pathway in patients with cancer. Transl Res. 2016; 167:61-6.

32. Eskelinen EL. The dual role of autophagy in cancer. Curr Opin Pharmacol. 2011; 11:294-300.

33. Green DR, Reed JC. Mitochondria and apoptosis. Science. 1998; 281:1309-12.

34. Kannan K, Jain SK. Oxidative stress and apoptosis. Pathophysiology. 2000; 7:153-163.

35. Kalyanaraman B. Teaching the basics of cancer metabolism: Developing antitumor strategies by exploiting the differences between normal and cancer cell metabolism. Redox Biol. 2017; 12:833-842.

36. Rawlings JS, Rosler KM, Harrison DA. The JAK/STAT signaling pathway. J Cell Sci. 2004; 117:1281-1283.

37. Derenzini E, Lemoine M, Buglio D, Katayama H, Ji Y, Davis RE, Sen S, Younes A. The JAK inhibitor AZD1480 regulates proliferation and immunity in Hodgkin lymphoma. Blood Cancer J. 2011; 1:e46. Erratum in: Blood Cancer J. 2014; 4:e231.

38. Harrison C, Verstovsek S, McMullin MF, Mesa R. Janus kinase inhibition and its effect upon the therapeutic landscape for myelofibrosis: from palliation to cure? $\mathrm{Br} \mathrm{J}$ Haematol. 2012; 157:426-37.

39. Bhagwat N, Koppikar P, Keller M, Marubayashi S, Shank K, Rampal R, Qi J, Kleppe M, Patel HJ, Shah SK, Taldone T, Bradner JE, Chiosis G, Levine RL. Improved targeting of JAK2 leads to increased therapeutic efficacy in myeloproliferative neoplasms. Blood. 2014; 123:2075-83.

40. Fiskus W, Verstovsek S, Manshouri T, Rao R, Balusu R, Venkannagari S, Rao NN, Ha K, Smith JE, Hembruff SL, Abhyankar S, McGuirk J, Bhalla KN. Heat shock protein 90 inhibitor is synergistic with JAK2 inhibitor and overcomes resistance to JAK2-TKI in human myeloproliferative neoplasm cells. Clin Cancer Res. 2011; 17:7347-58.

41. LaFave LM, Levine RL. JAK2 the future: therapeutic strategies for JAK-dependent malignancies. Trends Pharmacol Sci. 2012; 33:574-82.

42. Wang Y, Fiskus W, Chong DG, Buckley KM, Natarajan K, Rao R, Joshi A, Balusu R, Koul S, Chen J, Savoie A, Ustun C, Jillella AP, et al. Cotreatment with panobinostat and JAK2 inhibitor TG101209 attenuates JAK2V617F levels and signaling and exerts synergistic cytotoxic effects against human myeloproliferative neoplastic cells. Blood. 2009; 114:5024-33.

43. Thomas SJ, Snowden JA, Zeidler MP, Danson SJ. The role of JAK/STAT signalling in the pathogenesis, prognosis and treatment of solid tumours. Br J Cancer. 2015; 113:365-371. 
44. Hao Y, Chapuy B, Monti S, Sun HH, Rodig SJ, Shipp MA. Selective JAK2 inhibition specifically decreases Hodgkin lymphoma and mediastinal large B cell lymphoma growth in vitro and in vivo. Clin Cancer Res. 2014; 20:2674-2683.

45. Scott LM, Gandhi MK. Deregulated JAK/STAT signalling in lymphomagenesis, and its implications for the development of new targeted therapies. Blood Rev. 2015; 29:405-415.

46. Fantin VR, Loboda A, Paweletz CP, Hendrickson RC, Pierce JW, Roth JA, Li L, Gooden F, Korenchuk S, Hou XS, Harrington EA, Randolph S, Reilly JF, et al. Constitutive activation of signal transducers and activators of transcription predicts vorinostat resistance in cutaneous T-cell lymphoma. Cancer Res. 2008; 68:3785-94.

47. Guthrie GJ, Roxburgh CS, Richards $\mathrm{CH}$, Horgan PG, McMillan DC. Circulating IL-6 concentrations link tumour necrosis and systemic and local inflammatory responses in patients un-dergoing resection for colorectal cancer. Br J Cancer. 2013; 109:131-7.

48. Camporeale A, Poli V. IL-6, IL-17 and STAT3: a holy trinity in auto-immunity? Front Biosci (Landmark Ed). 2012; 17:2306-26.

49. Valdhoen M. Interleukin 17 is a chief orchestrator of immunity. Nat Immunol. 2017; 18:612-621.
50. Bagca BG, Ozalp O, Kurt CC, Mutlu Z, Saydam G, Gunduz C, Avci CB. Ruxolitinib induces autophagy in chronic myeloid leukemia cells. Tumour Biol. 2016; 37:1573-9.

51. Ojha R, Singh SK, Bhattacharyya S. JAK-mediated autophagy regulates stemness and cell survival in cisplatin resistant bladder cancer cells. Biochim Biophys Acta. 2016; 1860:2484-97.

52. Rodríguez-Enríquez S, Gallardo-Pérez JC, MarínHernández A, Aguilar-Ponce JL, Mandujano-Tinoco EA, Meneses A, Moreno-Sánchez R. Oxidative phosphorylation as a target to arrest malignant neoplasias. Curr Med Chem. 2011; 18:3156-67.

53. Chou TC, Talalay P. Quantitative analysis of dose-effect relationships: the combined effects of multiple drugs or enzyme inhibitors. Advances In Enzyme Regulation. 1984; 22:27-55.

54. Chou TC. Theoretical basis, experimental design, and computerized simulation of synergism and antagonism in drug combination studies. Pharmacological Reviews. 2006; 58:621-681.

55. Chou TC. Preclinical versus clinical drug combination studies. Leukemia And Lymphoma. 2008; 49:2059-2080. 